\title{
Measuring mechanical anisotropy of the cornea with Brillouin microscopy
}

\author{
Amira M. Eltony ${ }^{1}$, Peng Shao ${ }^{1,+}$, and Seok-Hyun Yun ${ }^{1,2, *}$ \\ ${ }^{1}$ Harvard Medical School and Wellman Center for Photomedicine, Massachusetts General Hospital, Boston, MA, \\ 02114, USA \\ ${ }^{2}$ Harvard-MIT Health Sciences and Technology, Cambridge, MA, 02139, USA \\ +Present affiliation: Reveal Surgical Inc., Montréal, QC, H2N 1A4, Canada \\ *syun@hms.harvard.edu
}

\begin{abstract}
Load-bearing tissues are typically fortified by networks of protein fibers, often with preferential orientations. This fiber structure imparts the tissues with direction-dependent mechanical properties optimized to support specific external loads. To accurately model and predict tissues' mechanical response, it is essential to characterize the anisotropy on a microstructural scale. Previously, it has been difficult to measure the mechanical properties of intact tissues noninvasively. Here, we use Brillouin optical microscopy to visualize and quantify the anisotropic mechanical properties of corneal tissues at different length scales. We derive the stiffness tensor for a lamellar network of collagen fibrils and use angle-resolved Brillouin measurements to determine the longitudinal stiffness coefficients (longitudinal moduli) describing the ex vivo porcine cornea as a transverse isotropic material. Lastly, we observe significant mechanical anisotropy of the human cornea in vivo, highlighting the potential for clinical applications of off-axis Brillouin microscopy.
\end{abstract}

\section{Introduction}

Biological soft tissues are typically fiber-reinforced composites, consisting of a network of collagen and/or elastin fibers embedded in a hydrated matrix. In load-bearing regions, such as muscles, blood vessel walls, or the lining of the gastrointestinal tract, this microstructure imparts tissues with their unique combination of high flexibility and strength. Preferential fiber alignment is a natural consequence of the fiber structure conforming to resist the directional stresses and strains acting on a particular organ, resulting in anisotropic mechanical properties. Biaxial tensile testing has identified mechanical anisotropy in tissue samples excised from the lungs ${ }^{1,2}$, the pulmonary arteries ${ }^{3}$, the pericardium ${ }^{4}$, the aorta ${ }^{5}$, the retina ${ }^{6}$, the vagina ${ }^{7}$, and the $\operatorname{skin}^{8,9}$ among others. The anisotropic behavior of these tissues is fine-tuned for their specific physiological functions. As such, fibrillar remodeling, resulting in changing tissue anisotropy, is often an indication of disease. There has been sustained interest in characterizing the mechanical anisotropy of soft tissues, particularly at the microstructural scale, in order to build accurate constitutive models which can be used to predict the tissues' response to mechanical loading ${ }^{10}$.

Brillouin microscopy is an emerging optical technique for biomechanical characterization of cells and tissues ${ }^{11}$. The technique is based on inelastic light scattering from naturally-occurring (spontaneous) or stimulated acoustic waves in the material being probed. The resulting frequency shift of the scattered light, or the Brillouin frequency shift, is related to the compressional acoustic wave speed and hence the longitudinal elastic properties of the material. By employing a focused laser beam, Brillouin microscopy is able to map elastic properties with optical-scale spatial resolution $(\sim \mu \mathrm{m})$. Brillouin microscopy has been used to measure localized mechanical changes in the eyes of patients with the corneal disease keratoconus ${ }^{12}$, and 
depth-dependent mechanical changes induced by collagen crosslinking $(\mathrm{CXL})^{13}$. Brillouin scattering has revealed anisotropic properties of solid-state materials ${ }^{14}$, silks ${ }^{15}$, and plant stems ${ }^{16}$, but to our knowledge, has not yet been used to analyze soft biological tissue such as the cornea.

The cornea has anisotropic mechanical properties because of preferential fibril alignment tangential to the surface of the eye. The mechanical strength, shape, and transparency of the cornea stem from its microstructure: an intricate lattice of collagen fibrils embedded in a gel matrix. Characterizing corneal anisotropy is important in predicting the response of the cornea to different mechanical stimuli. Measuring the degree of anisotropy may also be a useful indicator of changes in the cornea's collagen organization due to disease or following surgical intervention ${ }^{17-19}$. Some evidence of corneal anisotropy in the porcine eye has been obtained using ultrasound supersonic shear wave imaging ${ }^{20}$, although with limited sensitivity and spatial resolution. Uniaxial tensile testing ${ }^{21}$ and optical coherence elastography ${ }^{22}$ have also been applied to the cornea, but were limited to comparing elasticity in tangential directions.

In this work, we directly visualize the alternating elastic properties of crisscrossing fiber layers (lamellae) in the porcine cornea using a high-resolution Brillouin microscope and confirm the interpretation of this pattern using transmission electron microscopy (TEM). We then measure the corresponding mechanical anisotropy in longitudinal modulus of the bulk porcine cornea using Brillouin microscopy which we find to be consistent with the microstructural-scale imaging. We derive a composite model for the lamellar network of collagen fibrils in the cornea and use our angle-resolved Brillouin measurements to determine the stiffness coefficients (longitudinal moduli) describing the transverse isotropic tissue. Finally, we analyze the angle-dependent Brillouin corneal maps of healthy human subjects in vivo and determine the mechanical anisotropy of the normal human cornea.

\section{Results}

\section{Composite model of corneal anisotropy}

The stroma, which makes up the bulk of the cornea and contributes most to its biomechanical properties, is organized into layers approximately 2-3 $\mu \mathrm{m}$ thick called lamellae. Within each lamella, collagen fibrils of $\sim 25 \mathrm{~nm}$-diameter are co-aligned tangentially to the corneal surface ${ }^{23}$. X-ray diffraction measurements suggest that collagen fibrils do not form a perfect crystalline lattice, but instead exhibit short-range order ${ }^{24}$. However, for the purpose of modeling, the fibril organization within an individual lamella can be well approximated by a pseudo-hexagonal lattice ${ }^{25}$. X-ray diffraction studies have established that the fibril diameter is $31 \mathrm{~nm}$ (humans) and $37 \mathrm{~nm}$ (pigs) and the interfibrillar Bragg spacing is $55 \mathrm{~nm}$ (humans) and $59 \mathrm{~nm}$ (pigs), corresponding to a fibril volume fraction $V^{(f)}$ of 0.22 (humans) and 0.28 (pigs) ${ }^{25}$.

A single corneal lamella can be modelled as an aligned fiber composite, with the 1-direction oriented parallel to the fiber axis, and the 2- and 3-directions orthogonal to it (Fig. 1a). Since a collagen fibril can be assumed to be radially symmetric, it

has transverse isotropic symmetry, and its stiffness tensor, $\boldsymbol{C}^{(f)}$, has five independent coefficients (see Supplementary Materials). Likewise, the gel matrix surrounding the fibrils can also be described with a stiffness tensor, $\boldsymbol{C}^{(m)}$. Assuming the fibers do not 
slip within the matrix under 1-directional loading (isostrain) and the stress is distributed equally across the fibers and matrix during 2- and 3-directional loading (isostress), the stiffness coefficients along the 1,2, and 3 directions, or longitudinal moduli, of a single lamella (Fig. 1b) can be expressed as a function of the individual moduli of the fibers $(f)$ and of the extrafibrillar matrix $(m)$ (law of mixtures): $C_{11}^{(\text {lamella) }}=C_{11}^{(f)} V^{(f)}+C_{11}^{(m)}\left(1-V^{(f)}\right), \frac{1}{C_{22}^{\text {(lamella) }}}=\frac{V^{(f)}}{C_{22}^{(f)}}+\frac{1-V^{(f)}}{C_{22}^{(m)}}, \frac{1}{C_{33}^{(\text {lamella) }}}=\frac{V^{(f)}}{C_{33}^{(f)}}+\frac{1-V^{(f)}}{C_{33}^{(m)}}$, where $C_{22}^{(m)}=C_{33}^{(m)}$ and $C_{22}^{(f)}=C_{33}^{(f)}$ by symmetry. An individual lamella is thus transverse isotropic with plane of symmetry 2-3 (i.e. orthogonal to the fibril axis).

The corneal stroma is composed of a stack of 300-500 lamellae of varying orientations. The axes of the collagen fibrils in successive lamellae are not truly random, but tend to lie along orthogonal meridians in the medial-lateral and superior-inferior directions, particularly in the posterior cornea ${ }^{24}$. For simplicity, we model the stroma as a stack of layers with half oriented in the medial-lateral direction and half in the superior-inferior direction, which yields:

$$
\begin{aligned}
C_{x x}^{\text {(stroma) }}=C_{y y}^{\text {(stroma) }} & =\frac{1}{2} C_{11}^{(\text {lamella })}+\frac{1}{2} C_{33}^{\text {(lamella })} \\
C_{z z}^{\text {(stroma })} & =C_{22}^{\text {(lamella) }}=C_{33}^{\text {(lamella })}
\end{aligned}
$$

Here, the $(x, y, z)$ coordinate system is defined in real space such that the $z$-direction is orthogonal to the cornea, and the $x$-and $y$-directions are tangential (medial-lateral and superior-inferior, Fig. 1b). In this model, the cornea is transverse isotropic with plane of symmetry $x-y$ (i.e. tangential to the cornea).

For backward Brillouin light scattering spectroscopy with probe light entering the corneal tissue at an angle $\theta$ to the optic axis (Fig. 1c), the Brillouin frequency shift $\Omega$ is given by: $\Omega(\theta)=\frac{2 n(\theta) v(\theta)}{\lambda}=\frac{2 n(\theta)}{\lambda} \sqrt{\frac{C(\theta)}{\rho}}$, where $n(\theta)$ is the index of refraction of the material, $\lambda$ is the wavelength of light, $v(\theta)=\sqrt{C(\theta) / \rho}$ is the longitudinal-wave acoustic speed, and $C(\theta)$ is the effective longitudinal modulus of the stroma at angle $\theta$ to normal. For simplicity, we consider a case where the incident beam is in the $x-z$ plane. The formalism described below can be applied to other tilt directions with appropriate coordinate transformations.

The index of refraction in the cornea is known to differ slightly depending on the direction of light propagation. Mueller matrix ellipsometry of the human cornea in vivo found that $n_{x}-n_{z}=1.4 \times 10^{-4},{ }^{26}$ similar to other measurements ${ }^{27}$ ). If individual lamellae are modelled as uniaxial birefringent layers stacked orthogonally, then $n_{x}-n_{z}=2.8 \times 10^{-4}$ theoretically for a single lamella ${ }^{28}$. As we will see, the contribution of this magnitude of birefringence to the Brillouin shift is 2-3 orders of magnitude lower than that of mechanical anisotropy. Therefore, we can neglect the birefringence and assume $n(\theta)$ to be a constant independent of $\theta$.

An exact analytic expression for $C(\theta)$ can be written in terms of the stiffness tensor coefficients (Supplementary Materials). In the case of weak transverse isotropy, i.e. $C_{x x}^{\text {(stroma) }} \approx C_{z z}^{\text {(stroma) }}$, the effective longitudinal modulus can be expressed (Supplementary Materials) as: $C(\theta) \approx C_{z z}+\left(C_{x x}-C_{z z}\right) \sin ^{4} \theta+2\left(C_{x z}+2 G_{y z}-C_{z z}\right) \sin ^{2} \theta \cos ^{2} \theta$, where the superscripts were ignored, and $G_{y z}\left(\ll C_{z z}\right)$ is shear modulus of the stroma in the $y-z$ plane. We confirm $C(0)=C_{z z}$, and $C(\pi / 2)=C_{x x}$ (the beam 
is tilted toward the $x$-axis). The difference of these two values characterizes the magnitude of anisotropy of the tissue. We introduce anisotropic parameters, $\alpha_{x x}$ and $\delta$, defined as follows:

$$
\begin{aligned}
& \alpha_{x x}=C_{x x} / C_{z z}-1 \\
& \delta=\left(C_{x z}+2 G_{y z}\right) / C_{z z}-1
\end{aligned}
$$

Thus, we can write

$$
C(\theta)=C_{z z}\left(1+\alpha_{x x} \sin ^{4} \theta+2 \delta \sin ^{2} \theta \cos ^{2} \theta\right)
$$

The anisotropic coefficients are analogous, but not identical, to the Thomson parameters used to describe seismic anisotropy (Note: Thomson parameters are defined in terms of wave speeds rather than elastic moduli) ${ }^{29}$.

Putting Eq. 1 into Eq. 2, we find:

$$
\alpha_{x x}^{(\text {stroma })}=\frac{\left(C_{11}^{(\text {lamella })}+C_{33}^{(\text {lamella })}\right) / 2}{C_{33}^{(\text {lamella })}}-1=\frac{1}{2} \alpha_{11}^{(\text {lamella })}
$$

Here $\alpha_{11}^{(\text {lamella) }}$ corresponds to the anisotropic parameter of a single lamella (with weak anisotropy $C_{33}^{(\text {lamella) }} \approx C_{11}^{(\text {lamella) }}$ ). It can be further shown that

$$
\alpha_{11}^{(\text {lamella) }}=\left[\beta_{2} V^{(f)}+\beta_{1}\left(1-V^{(f)}\right)\right]\left[\beta_{1} V^{(f)}+1-V^{(f)}\right] / \beta_{1}-1
$$

where $\beta_{1}=C_{33}^{(f)} / C_{11}^{(m)}$ and $\beta_{2}=C_{33}^{(f)} / C_{11}^{(f)}$ (Supplementary Materials).

\section{Brillouin imaging of individual corneal lamellae}

To test if Brillouin microscopy can indeed detect the anisotropy of individual lamellae, we excised the cornea from a porcine eye within 4 hours of sacrifice. First, the corneal flap was measured en-face and imaged using an inverted confocal Brillouin microscope (Fig. 2a). In this orientation, the laser is orthogonal to almost all collagen fibrils, so we expect the lamellar contrast to disappear. Indeed, the resulting $38 \mu \mathrm{m} \times 38 \mu \mathrm{m}$ Brillouin image is mostly uniform with a Brillouin value of $7.981 \pm 0.078$ $\mathrm{GHz}$ (mean \pm standard deviation, measured at $532 \mathrm{~nm}$ ). Second, we cut the corneal flap across the middle and mounted it vertically, allowing the cross-section to be imaged from beneath using an inverted confocal Brillouin microscope with a lateral resolution of $\sim 0.5 \mu \mathrm{m}$ (Fig. 2 c). Stromal tissue with a cross-sectional area of $30 \mu \mathrm{m} \times 30 \mu \mathrm{m}$ located in the center and the middle third depth-wise was imaged (Fig. 2d). Lamellae in the middle third of the corneal stroma are predominantly orthogonally stacked with little interleaving, resulting in alternating layers of higher and lower elastic modulus (from $C_{11}^{(\text {lamella) }}$ to $C_{33}^{(\text {lamella) }}$ ). This pattern is evident in the Brillouin images which show ribbons of width 0.5 - $5 \mu \mathrm{m}$ with alternating Brillouin values.

After identifying oriented lamella of lowest and highest Brillouin values (see Methods), we estimate that $\Omega(0)=8.032 \pm$ 
$0.008 \mathrm{GHz}$ and $\Omega(\pi / 2)=8.879 \pm 0.018 \mathrm{GHz}$. This inter-lamellar contrast of $10.5 \%$ in Brillouin values (i.e. $\Omega(\pi / 2) / \Omega(0)=$ 1.105) corresponds to $\alpha_{x x}^{(\text {lamella })}=C_{x x} / C_{z z}-1=(\Omega(\pi / 2) / \Omega(0))^{2}-1=0.222$. We expect the value of $\Omega(0)$ from the crosssection to approximately match the average value in the en-face image, which also measures $\Omega(0)$. They are indeed similar for this cornea $(8.032 \mathrm{GHz}$ and $7.981 \mathrm{GHz}$, respectively) with the slight difference likely corresponding to spatial variation as these could not be measured at the exact same location.

Figure 2e-f shows TEM images of a single corneal cross-section taken in a similar region (central area of cornea, middle of stromal depth) at two different magnifications. Layers of alternating fibril orientation are visible, as expected from textbook examples $^{30}$. This lamellar structure is consistent with what we observed in the Brillouin images.

We made further measurements using a confocal Brillouin microscope with a $780 \mathrm{~nm}$ laser, observing the same dramatic contrast due to corneal anisotropy. Fig. 3a shows the $28 \mu \mathrm{m} \times 28 \mu \mathrm{m}$ en-face image of a (different) cornea, with Brillouin value $5.434 \pm 0.012 \mathrm{GHz}$ (mean \pm standard deviation, consistent with the wavelength-dependence of the Brillouin shift). Fig. 3b shows cross-sectional images of the same cornea at three different (relative) depths. After identifying oriented lamella of lowest and highest Brillouin values as before, we estimate that $\Omega(0)=5.395 \pm 0.002 \mathrm{GHz}$ and $\Omega(\pi / 2)=5.916 \pm 0.008 \mathrm{GHz}$ for this cornea, corresponding to $\alpha_{x x}^{\text {(lamella) }}=0.202$. Again, we expect the value of $\Omega(0)$ from the cross-section to approximately match the average value in the en-face image, which also measures $\Omega(0)$. They are indeed similar for this cornea (5.395 $\mathrm{GHz}$ and $5.434 \mathrm{GHz}$, respectively).

Fig. 3c shows a larger $(37 \mu \mathrm{m} \times 37 \mu \mathrm{m})$ cross-sectional Brillouin image of a second cornea (from a different animal). For this sample, we estimate that $\Omega(0)=5.350 \pm 0.003 \mathrm{GHz}$ and $\Omega(\pi / 2)=5.798 \pm 0.004 \mathrm{GHz}$, corresponding to $\alpha_{x x}^{(\text {lamella })}=0.174$. Fig. 3d-e show Brillouin images of a third cornea (from another pig) measured en-face and in cross-section, respectively, at three different (relative) depths. In the en face images, the Brillouin value remains fairly uniform throughout the depth with an average value of $\Omega(0)=5.468 \pm 0.007 \mathrm{GHz}$. In the cross-sectional images, the lamellar pattern is evident with estimated $\Omega(0)=5.459 \pm 0.001 \mathrm{GHz}$ and $\Omega(\pi / 2)=5.988 \pm 0.009 \mathrm{GHz}$, corresponding to $\alpha_{x x}^{\text {(lamella) }}=0.203$.

We collected a total of 31 cross-sectional Brillouin images from 7 ex vivo porcine corneas of 7 pigs using the $780 \mathrm{~nm}$ Brillouin microscope. The inter-lamellar difference in the Brillouin frequency varied between corneas, perhaps due to mechanical variation from animal to animal, or variation from location to location in the cornea. Variation may also be attributed to differences in hydration level (despite our best efforts; see Methods) and differences in the orientation of the cross-sections or, in other words, the orientation of collagen fibrils with respect to the optical beam. We found that $\Omega(0)$ ranged from 5.310 to $5.461 \mathrm{GHz}$, and $\Omega(\pi / 2)$ ranged from 5.636 to $6.098 \mathrm{GHz}$. The resulting $\alpha_{x x}^{\text {(lamella) }}$ values ranged from 0.126 to 0.247 with mean value $0.184 \pm 0.031$. The distribution of $\alpha_{x x}^{\text {(lamella) }}$ values for all measurements is shown in Fig. 3f.

\section{Angle-dependence of bulk cornea Brillouin measurements}

The layered structure observed at the microscopic scale translates to a weak anisotropy of the bulk stroma, which we expected to see in larger-scale Brillouin measurements. For measurements of the intact cornea, we used a lower resolution (numerical aperture of $\sim 0.1)$, near-infrared $(\lambda=780 \mathrm{~nm})$ Brillouin imaging system ${ }^{31}$. As before, corneal flaps were excised from porcine 
eye globes received within 4 hours of sacrifice. Intact corneal flaps were mounted in a Barron chamber which could be rotated, allowing for different laser incidence angles at a specific location on the cornea (Fig. 4a). Brillouin measurements of 11 cornea samples were made at 6 different laser incidence angles in either the central cornea or an off-center location.

Results are shown in Fig. 4b (central location) and Fig. 4c (off-center locations). Angles in air were converted to angles in tissue assuming a stromal index of refraction of $1.376 .{ }^{32}$ The maximum possible angle is limited by beam aberration or refraction at larger angles. Angle-dependence data were fit to the weak transverse isotropy model (Eq. 3), with free parameters $\Omega(0), \alpha_{x x}^{\text {(stroma) }}$, and $\delta$. Agreement with the model was fairly good, with $R^{2}$ ranging from 0.74 to 0.96 for the different samples. The fitting results were similar when $\Omega(0)$ was removed as a free parameter. Values and confidence ranges for all fitted parameters are tabulated in the Supplementary Materials, Table S1.

Although there was variation from one cornea to another, we did not observe a significant difference in the average fitted anisotropic parameter with $\alpha_{x x}^{\text {(stroma) }}=0.108 \pm 0.020$ in the central cornea, and $\alpha_{x x}^{\text {(stroma) }}=0.110 \pm 0.035$ in other locations. From the cross-sectional imaging experiment described in the previous section, we have measured $\alpha_{x x}^{\text {(lamella) }}$ ranging from 0.126 to 0.247 with mean value $0.184 \pm 0.031$. Based on Eq. 4 , we expect $\alpha_{x x}^{\text {(stroma) }}$ to correspondingly range from $\sim 0.063$ to 0.123 with mean value $0.092 \pm 0.015$. This is fairly similar to $\alpha_{x x}^{\text {(stroma) }}=0.109 \pm 0.030$ (average at all locations), as determined from the angle-resolved measurements here.

\section{Anisotropy in vivo in Brillouin maps of the human cornea}

We also found evidence of corneal anisotropy in Brillouin measurements of human subjects. Study participants with normal corneas $(n=4,3: 1 \mathrm{M}: \mathrm{F}, 31.5 \pm 2.4 \mathrm{Y} / \mathrm{O})$ were scanned using a custom-built Brillouin imaging system at a wavelength of $780 \mathrm{~nm}^{31}$. When a human subject is imaged using this instrument, the subject's gaze angle is directed towards a stationary fixation target while the Brillouin interface is translated right-left and up-down to measure different locations laterally across the cornea. Because the optical path is rigidly fixed with respect to the interface, as the laser position changes, the angle of incidence (relative to the corneal surface) also changes. When the laser incidence angle is close to $0^{\circ}$, this change is small, but for larger tilt angles (corresponding to a fixation target at $\sim 20^{\circ}$ ) there is a gradually increasing angle of incidence as the laser moves from right to left across the cornea (see diagrams in Fig. 5a-b).

Data from approximately 30 axial scans were combined to create a color-coded map of the mean Brillouin shift laterally across the corneal stroma. The irregular shape of the maps is owing to manual positioning of the measurement points using a slit-lamp joystick. An increasing Brillouin gradient from right to left is apparent in the resulting maps, with larger Brillouin values for larger incidence angles as we would expect. The Brillouin maps of three different human subjects displaying this gradient are shown in Fig. 5b. By contrast, when the laser angle is changed to minimize the variation in incidence angle (Fig. 5a), the resulting Brillouin map is more uniform with no obvious gradient.

To analyze the angle-dependence inherent in the human maps, we converted the map coordinates to approximate angles assuming a spherical corneal surface with $7.8 \mathrm{~mm}$ radius-of-curvature ${ }^{30}$ and fit to the transverse isotropy model (Eq. 3 ). The fitted anisotropic parameter values for the human corneas measured with larger tilt angles are $\alpha_{x x}^{(\text {stroma) }}=0.064 \pm 0.065$, 
$\alpha_{x x}^{\text {(stroma) }}=0.070 \pm 0.012, \alpha_{x x}^{\text {(stroma) }}=0.052 \pm 0.010$ (left to right). Agreement with the model was not as good as in the porcine case (previous section), with $R^{2}$ ranging from 0.46 to 0.60 for these 3 subjects, perhaps because of the relatively crude angle estimation and generally greater variability in the data. For the cornea measured with laser incidence angle close to $0^{\circ}, \alpha_{x x}^{\text {(stroma) }}=0.027 \pm 0.021$, but the fit quality was poor $\left(R^{2}=0.09\right)$, likely due to the small range of angles. Values and confidence ranges for all fitted parameters are tabulated in the Supplementary Materials, Table S3.

\section{Anisotropy in Brillouin maps of the porcine cornea}

Similar to the human measurements in the previous section, we also created Brillouin maps of porcine corneas and analyzed the angle-dependence. Intact porcine eye globes received within 4 hours of sacrifice were scanned using a custom-built Brillouin imaging system at a wavelength of $780 \mathrm{~nm}^{31}$. Data from a $7 \times 7$ grid of axial scans were combined to create a color-coded map of the mean Brillouin shift laterally across the corneal stroma.

Fig. 6a shows the relatively uniform Brillouin map of a porcine cornea scanned with the laser approximately normal to the corneal apex and less variation in incidence angle across the cornea. Fig. $6 \mathrm{~b}$ (leftmost) shows the Brillouin map of the same cornea scanned with the laser tilted by $\sim 15^{\circ}$ (with respect to the optical axis) causing greater variation in incidence angle across the cornea, resulting in an increasing Brillouin gradient from right to left. A similar gradient is apparent in the right four Brillouin maps in Fig. 6b, which correspond to the corneas of four other animals also measured with larger tilt angles.

As for the human maps, we converted the map coordinates to angles in order to analyze the Brillouin angle-dependence (Fig. 6c-d). Because the porcine corneas were automatically scanned (unlike the human maps which were taken manually), we were able to use the known coordinates of the corneal surface to compute the individual corneal radii of curvature and more accurately calculate the local incidence angles. The corneal radii of curvature ranged from $7.61 \mathrm{~mm}$ to $8.79 \mathrm{~mm}$ with average $8.33 \pm 0.44 \mathrm{~mm}$.

Data were fit to the weak transverse isotropy model (Eq. 3) as in previous sections. Agreement with the model was fairly good, with $R^{2}$ ranging from 0.78 to 0.84 for the different samples. Although the angle-dependence data shown in Fig. 6e and in $6 \mathrm{f}$ (leftmost) were measured in the same cornea, there is a discrepancy between the fitted values of $\alpha_{x x}^{\text {(stroma) }}=0.120 \pm 0.010$ and $\alpha_{x x}^{\text {(stroma) }}=0.082 \pm 0.005$ (respectively), perhaps owing to the small range of angles in Fig. 6e. Values and confidence ranges for all fitted parameters are tabulated in the Supplementary Materials, Table S2.

For the five corneas shown in Fig. 6f, fitted values of $\alpha_{x x}^{\text {(stroma) }}$ ranged from 0.075 to 0.122 with average $0.096 \pm 0.018$. This value is similar to the fitted value $\alpha_{x x}^{\text {(stroma) }}=0.109 \pm 0.030$ obtained via angle-resolved measurements of the bulk porcine cornea and the estimated value $\alpha_{x x}^{\text {(stroma) }}=0.092 \pm 0.015$ from Brillouin imaging of individual corneal lamellae.

\section{Longitudinal stiffness coefficients of the cornea}

From the angle-resolved Brillouin measurements of the porcine and human corneas we can estimate the longitudinal moduli.

Data were fit to the weak transverse isotropy model (Eq. 3), yielding fitted values for $\Omega(0)=\frac{2 n}{\lambda} \sqrt{C_{z z} / \rho}, \alpha_{x x}=C_{x x} / C_{z z}-1$,

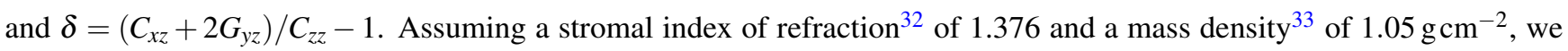


extracted all three longitudinal moduli $C_{z z}$ and $C_{x x}=C_{y y}$ for each sample as well as the combination of moduli $C_{x z}+2 G_{y z}$ $\left(\approx C_{x z}\right)$ (Table 1). The symmetry of transverse isotropy dictates that $C_{y z}=C_{x z}$ and $C_{x y}=C_{x x}-2 G_{x y} \approx C_{x x}$. Therefore, all the longitudinal stiffness coefficients $C_{i j}$ 's $(i, j=x, y, z)$ have been determined.

It is interesting to compare the two species. The human corneas exhibited greater out-of-plane stiffness $C_{z z}$ than the porcine corneas. The in-plane stiffness, $C_{x x}$ and $C_{y y}$, however, was similar between human and porcine corneas. As a result, the human corneas have a lower degree of anisotropy than the porcine corneas.

The anisotropic parameters we determined from measurements of porcine and human corneas can be used in our composite model to estimate the properties of the cornea's constituent parts: collagen fibrils and gel matrix. The gel matrix in particular has not previously been accessible for material characterization. The mean anisotropic parameter measured in humans was $\alpha_{x x}^{\text {(stroma) }}=0.062 \pm 0.008$ (3 subjects in Fig. 5f), corresponding to $\alpha_{x x}^{\text {(lamella) }}$ (human) $=0.124 \pm 0.015$ (Supplementary Materials). For porcine samples, we measured $\alpha_{x x}^{(\text {lamella) }}$ (porcine) $=0.184 \pm 0.031$ (cross-sectional data in Fig. 2f). Considering the different fibril volume fractions ${ }^{25}, V^{(f)}($ human $)=0.22$ and $V^{(f)}$ (porcine) $=0.28$, and assuming that the material properties of the collagen fibrils and gel matrix are similar between humans and pigs (specifically, $\beta_{1}, \beta_{2}$ values within 5\%), we can estimate the ratio of the longitudinal modulus in the axial ' 1 ' direction (along the fiber) to that in the transverse ' 2,3 ' directions (orthogonal to the fiber), $\beta_{2}$ (human) $=C_{11}^{(f)} / C_{33}^{(f)}=1.35$ and $\beta_{2}$ (porcine) $=1.41$ (Eq. 5). Similar values have been reported in ultrasound measurements of collagen from the bovine Achilles tendon which found $C_{11}^{(f)} / C_{33}^{(f)}=1.47$ (fixed $)^{34}, C_{11}^{(f)} / C_{33}^{(f)}=1.33$ $(\text { fresh })^{35}$. Likewise, we estimate that the ratio of the longitudinal modulus of the gel matrix (assumed isotropic) to that of the fiber (in the transverse direction) is $\beta_{1}$ (human) $=C_{11}^{(m)} / C_{33}^{(f)}=0.70$ and $\beta_{1}$ (porcine) $=0.68$, suggesting that the mechanical contrast between matrix and fibers is relatively low in the transverse direction.

\begin{tabular}{|c|c|c|}
\hline Stiffness coefficients & Porcine ex vivo (GPa) & Human in vivo $(\mathrm{GPa})$ \\
\hline$C_{z z}$ & $2.567 \pm 0.012$ & $2.738 \pm 0.001$ \\
$C_{x x}, C_{y y}$ & $2.848 \pm 0.078$ & $2.908 \pm 0.022$ \\
$C_{x z}+2 G_{y z}$ & $2.593 \pm 0.024$ & $2.789 \pm 0.010$ \\
\hline
\end{tabular}

Table 1. Longitudinal elastic moduli for porcine corneas ex vivo (at $\sim 23^{\circ} \mathrm{C}$ ) and human corneas in vivo measured by angle-resolved Brillouin measurements $(\lambda=780 \mathrm{~nm})$. Mean \pm standard deviation from 4 samples in each group.

\section{Discussion}

We have demonstrated that individual stromal lamellae can be resolved based on their Brillouin shift contrast. This contrast arises directly from the anisotropic elastic properties of the lamellae stemming from their fibrillar organization. We derived a composite model describing the lamellar network of collagen fibrils in the cornea. In aggregate, the crisscrossing lamellae produce a weakly transverse isotropic stroma. We confirmed that the Brillouin frequency shift of the cornea is indeed angle-dependent. Consistent with our model, the anisotropic parameter $\alpha_{x x}^{\text {(stroma) }}=0.092 \pm 0.015$ estimated from Brillouin imaging of individual cornea lamellae agrees fairly well (within uncertainty ranges) with the values obtained via angle-resolved measurements 
at a single point, $\alpha_{x x}^{\text {(stroma) }}=0.109 \pm 0.030$, and via angle-resolved analysis of map data, $\alpha_{x x}^{\text {(stroma) }}=0.096 \pm 0.018$. While techniques such as TEM have previously elucidated the fibrillar architecture of the cornea, this work uniquely characterizes the elastic properties of the cornea at the microstructural scale, allowing us to estimate the stiffness coefficients (longitudinal moduli) describing the transverse isotropic tissue. This material information is useful for detailed numerical simulations of the cornea. Equally important, we were able to connect the properties at the micro-scale to those of the bulk cornea, a critical link for understanding the impact of diseases or surgeries that alter the collagen structure.

Fiber-reinforced composite materials are ubiquitous in the body, where their mechanical properties serve their physiological functions. For example, the tympanic membrane of the ear has a lamellar collagen structure similar to that of the cornea and is also mechanically anisotropic ${ }^{36}$. A challenge in reconstructive ear surgery is replicating the complex, anisotropic material properties of the tympanic membrane so that the frequency response and mode shapes required for hearing can be restored ${ }^{37}$. Recent studies have explored laser-induced collagen remodeling as a treatment for chronic inflammatory ear pathologies that aims to restore the intricate collagen network rather than replace it with a poorly-matched synthetic material ${ }^{38}$. The microstructure of the uterine cervix also contains an anisotropic network of collagen fibers ${ }^{39}$. Preterm birth is thought to be caused by premature weakening of the cervix under the increasing load of a growing fetus. Efforts are underway to better understand the collagen remodeling process that allows the cervix to become more pliable during gestation in order to devise prophylactics against preterm birth ${ }^{40}$. Likewise, arterial walls have a network of layered collagen and elastin fibers with a predominantly circumferential orientation ${ }^{41}$. Gradual collagen remodeling plays a major role in the weakening and rupture of abdominal aortic aneurysms, resulting in many deaths ${ }^{42}$. Current investigations into the structure-function relationships in soft tissues like these primarily rely on traditional mechanical techniques such as indentation or video extensometry. These techniques cannot probe the micro-scale elastic properties of the tissue, cannot typically probe all axes, and are often difficult to implement in vivo. Multi-angle (vector) Brillouin microscopy may find application in characterizing the anisotropic longitudinal elastic properties of fiber-composite tissues found throughout the body with high spatial resolution.

Another potential avenue of application is biomaterial design. Tissue engineered constructs must be able to closely mimic the in-vivo mechanical and structural properties of the tissues they aim to replace. Because many soft tissues exhibit intricate fiber networks with preferential fiber alignment, they typically have complex, anisotropic mechanical properties. This has been recognized and biomaterials that can imitate soft-tissue mechanical anisotropy have been a goal of tissue engineering for more than a decade $\mathrm{e}^{43-45}$ ). Brillouin microscopy could provide valuable material data for designing engineered biomaterials and then characterizing the resulting constructs.

Lastly, we observed significant directional dependence in human Brillouin maps measured in vivo. The $\sim 35 \mathrm{MHz}$ gradient over the angle of 0 to 40 deg is comparable to the magnitude of spatial variation detected in mild corneal disease ${ }^{31}$. Therefore, the angle dependence must be taken into account in the interpretation of raw measurement data. In principle, it can be removed by numerical correction or beam scanning with a constant incident angle.

This work has focused on anisotropy in longitudinal moduli because this is probed by current Brillouin microscopy. However, 
a complete description of the stiffness or compliance tensor of tissue also requires characterization of shear moduli. In particular, for soft tissues like the cornea, which is nearly incompressible, the shear moduli are used to model corneal deformation under an applied load such as inflation due to intraocular pressure. Unfortunately, at present there are no instruments measuring anisotropy in shear moduli in vivo directly, though developing technologies, such as optical coherence elastography, show promise $^{46}$. Recently, noncontact acoustic microtapping OCE detected large anisotropy in shear moduli in ex vivo porcine corneas $^{47,48}$. Because it measures longitudinal moduli, Brillouin microscopy is particularly sensitive to changes in molecular composition and concentration. In this work, Brillouin contrast arises due to the underlying fiber organization of the cornea. Hence, analyzing the angle-dependence or mechanical anisotropy may be a useful metric to detect disruption or remodeling of the regular collagen lattice due to disease or surgical intervention. We expect the Brillouin angle-dependence to decrease in degenerative conditions where the lamellar or fibrillar microstructure becomes disorganized. It would be interesting to measure the Brillouin angle-dependence in the cone region of a keratoconus cornea.

\section{Methods}

\section{Cross-sectional Brillouin measurements}

Porcine eye globes were received on ice within 4 hours of sacrifice and corneal flaps were immediately excised. A custom 3D-printed fixture was used to mount the corneal flap securely so that a clean slice could be made through the center of the cornea using a scalpel. The resulting slice was then sandwiched between two glass slides so that the cross-section abutted one edge of the slides. The slides and cornea were then mounted vertically in a custom-made holder atop a glass-bottom dish so that the corneal cross-section was optically accessible from below. Empty space in the glass bottom dish and between the slides was filled with corneal preservation medium (Alchimia S.r.l., Padua, Italy) to prevent drying of the corneal slice. The corneal images

in Fig. 2 were obtained using a confocal Brillouin microscope with a $532 \mathrm{~nm}$ single-frequency laser (Laser Quantum, U.K.) ${ }^{49}$. The corneal images in Fig. 3 were obtained using a second confocal Brillouin microscope with a $780 \mathrm{~nm}$ single-frequency laser (TOPTICA Photonics AG, Germany) and a virtually imaged phased array (VIPA) spectrometer. A co-aligned bright-field microscope was used to navigate to the middle region of the corneal depth for measurement. The Brillouin measurement volume per point was approximately $0.5 \mu \mathrm{m} \times 0.5 \mu \mathrm{m} \times 1.0 \mu \mathrm{m}$ and the integration time used was $150-250 \mathrm{~ms}$. Oriented corneal lamellae were identified in the resulting Brillouin image via line-by-line peak/trough detection. Within these regions, the Brillouin values of the upper/lower 10\% (respectively) were assumed to correspond to the extremes of fibrillar orientation (in-plane vs. out-of-plane) and averaged to estimate the degree of corneal anisotropy. For TEM analysis, a corneal slice, prepared as above, was fixed using K2 buffer and later imaged using a transmission electron microscope (model CM10, Philips Electron Optics, Eindhoven, The Netherlands).

\section{Brillouin angle-dependence measurements}

Corneal flaps with a 2-3 mm scleral ring were excised from porcine eye globes using a pair of curved scissors and placed in Carry-C corneal preservation medium (Alchimia S.r.l., Padua, Italy) at room temperature $\left(22-24{ }^{\circ} \mathrm{C}\right)$. To minimize perturbation 
of the cornea, the iris was not forcibly removed but was instead left attached to the corneal flap. For imaging, the intact corneal flap was mounted in a Barron chamber (Katena Products Inc., New Jersey, USA) filled with Carry-C corneal preservation medium (Alchimia S.r.l., Padua, Italy), which set an effective intraocular pressure (IOP) of about $15 \mathrm{mmHg}$, confirmed using a standard water column. For these measurements we used a different Brillouin instrument designed for whole-cornea imaging in vivo, at a wavelength of $780 \mathrm{~nm}$. This instrument has been described previously ${ }^{31}$ but was upgraded before these measurements to incorporate a $\mathrm{Rb}$ vapor cell before the spectrometer to remove elastically scattered light from the Brillouin scattered light for improved spectral extinction. The Barron chamber was mounted on a rotation stage (RP01, Thorlabs Inc., New Jersey, USA) so that the laser incidence angle could be adjusted. A small ink mark was used as a target to insure that the measurement point remained fixed on the corneal apex as the was angle varied. Because of refraction at the air-cornea interface, we expect the region of tissue being probed at different angles to be slightly offset. Assuming that the properties of the tissue are mostly uniform within a local area, we don't expect this to have a significant effect on the results. The Brillouin measurement volume per point was approximately $2 \mu \mathrm{m} \times 2 \mu \mathrm{m} \times 30 \mu \mathrm{m}$ and the integration time used ranged from 200-700 ms. The Brillouin value at each angle is obtained by averaging points measured throughout the stromal depth. To minimize the impact of environmental drifts, the cycle of angle measurements was repeated six times (alternating increasing and decreasing angle) and the resulting Brillouin values at each angle were averaged.

\section{Brillouin maps of human subjects}

Healthy subjects with normal corneas $(n=4,3: 1 \mathrm{M}: \mathrm{F}, 31.5 \pm 2.4 \mathrm{Y} / \mathrm{O})$ were imaged at two locations: the Institute for Refractive and Ophthalmic Surgery (IROC) in Zürich, Switzerland, and the Woolfson Eye Institute in Atlanta, Georgia, following approval from the Institutional Review Board (IRB) of Partners HealthCare, the Partners Human Research Committee (PHRC), the Institutional Review Board of IROC, Zürich, and the Woolfson Eye Institute Ethics Committee. Informed consent was obtained from every subject before imaging and all experiments were performed in accordance with the principles of the Declaration of Helsinki. Subjects were not compensated for their participation. In this study, a 'normal cornea' was classified as: less than 3 diopters refractive error, corneal thickness between 495 and $600 \mu \mathrm{m}$, normal corneal topography, no corneal pathology, and no history of eye disease. Study participants were scanned using a clinical Brillouin imaging system at a wavelength of $780 \mathrm{~nm}$ which has been described previously ${ }^{31}$. The system specifications are the same as the instrument used for porcine angle-dependence measurements but in a mobile unit. For human subjects, axial scans were taken at $\sim 30$ different locations across the cornea with an integration time of $300 \mathrm{~ms}$ per point, or $12 \mathrm{~s}$ per scan. Brillouin shift values were averaged through the stromal depth, yielding a single Brillouin value per location. A color-coded Brillouin map was then created by 2-dimensional interpolation between these points.

\section{Brillouin maps of porcine corneas}

Intact porcine eye globes were mounted on an XYZ translation stage (X-XYZ-LSM025A, Zaber Technologies Inc., Vancouver, Canada) for Brillouin measurement with the same instrument used for porcine angle-dependence measurements. Similarly, the 
Brillouin measurement volume per point was approximately $2 \mu \mathrm{m} \times 2 \mu \mathrm{m} \times 30 \mu \mathrm{m}$ and the integration time used ranged from 200-700 ms. The sample was translated $1 \mathrm{~mm}$ at a time to create a $7 \times 7$ grid spanning $6 \mathrm{~mm} \times 6 \mathrm{~mm}$. At each (x, y)-coordinate, an axial Brillouin scan was taken and then the corneal surface was moistened with a few drops of Carry-C corneal preservation medium (Alchimia S.r.l., Padua, Italy) in order to prevent drying during the course of measurement. The Brillouin value at each $(\mathrm{x}, \mathrm{y})$-coordinate is then obtained by averaging points measured throughout the stromal depth.

\section{Statistical Analysis}

Data analysis was performed using custom software written in Python using the Spyder environment. The SciPy and Lmfit libraries were used for non-linear least-squares minimization and curve-fitting. The R-squared (coefficient of determination) was used as a measure of the global fit of the model and 95\% confidence bands are indicated in all plots. Tables of values and confidence intervals for each fitted parameter are included in the Supplementary Materials for all samples/subjects in this study.

\section{Data Availability}

The processed data that support the findings of this study are included in this published article and its Supplementary Materials. Raw data are available from the corresponding author upon reasonable request.

\section{Code Availability}

The custom software used for data acquisition and data analysis are available from the corresponding author upon reasonable request.

\section{References}

1. Vawter, D. L., Fung, Y. C. \& West, J. B. Elasticity of excised dog lung parenchyma. J. Appl. Physiol. 45, 261-269 (1978).

2. Vawter, D. L., Fung, Y. C. \& West, J. B. Constitutive Equation of Lung Tissue Elasticity. J. Biomech. Eng. 101, 38-45 (1979).

3. Debes, J. C. \& Fung, Y. C. Biaxial mechanics of excised canine pulmonary arteries. Am. J. Physiol. - Hear. Circ. Physiol. 269, H433-H442 (1995).

4. Lee, M. C., LeWinter, M. M. \& Freeman, G. Biaxial mechanical properties of the pericardium in normal and volume overload dogs. Am. J. Physiol. - Hear. Circ. Physiol. 18, H222-H230 (1985).

5. Zhou, J. \& Fung, Y. C. The degree of nonlinearity and anisotropy of blood vessel elasticity. Proc. Natl. Acad. Sci. 94, 14255-14260 (1997).

6. Chen, K., Rowley, A. P. \& Weiland, J. D. Elastic properties of porcine ocular posterior soft tissues. J. Biomed. Mater. Res. - Part A 93, 635-645 (2009). 
7. Martins, P. et al. Prediction of nonlinear elastic behaviour of vaginal tissue: experimental results and model formulation. Comput. Methods Biomech. Biomed. Eng. 13, 327-337 (2010).

8. Lanir, Y. \& Fung, Y. C. Two-dimensional mechanical properties of rabbit skin-I. Experimental system. J. Biomech. 7, 29-34 (1974).

9. Ní Annaidh, A., Bruyère, K., Destrade, M., Gilchrist, M. D. \& Otténio, M. Characterising the Anisotropic Mechanical Properties of Excised Human Skin Aisling. J. Mech. Behav. Biomed. Mater. 5, 139-148 (2012).

10. Holzapfel, G. A. Biomechanics of Soft Tissue. In Lemaitre, J. (ed.) Handbook of Materials Behavior Models, Volume III, Section 10.11, 1057-1071 (Academic Press, San Diego, CA, 2001).

11. Scarcelli, G. \& Yun, S. H. Confocal Brillouin microscopy for three-dimensional mechanical imaging. Nat. Photonics 2, 39-43 (2008).

12. Scarcelli, G., Besner, S., Pineda, R., Kalout, P. \& Yun, S. In Vivo Biomechanical Mapping of Normal and Keratoconus Corneas. JAMA Ophthalmol. 133, 480-482 (2015).

13. Scarcelli, G. et al. Brillouin Microscopy of Collagen Crosslinking: Noncontact Depth-Dependent Analysis of Corneal Elastic Modulus. Investig. Ophthalmol. Vis. Sci. 54, 1418-1425 (2013).

14. Koski, K. J., Akhenblit, P., McKiernan, K. \& Yarger, J. L. Non-invasive determination of the complete elastic moduli of spider silks. Nat. Mater. 12, 262-267 (2013).

15. Zhang, Y. et al. The elastic constants of rubrene determined by Brillouin scattering and density functional theory. Appl. Phys. Lett. 110 (2017).

16. Elsayad, K. et al. Mapping the subcellular mechanical properties of live cells in tissues with fluorescence emission-Brillouin imaging. Sci. Signal. 9, 1-13 (2016).

17. Meek, K. M. et al. Changes in Collagen Orientation and Distribution in Keratoconus Corneas. Investig. Ophthalmol. Vis. Sci. 46, 1948-1956 (2005).

18. Morishige, N. et al. Second-Harmonic Imaging Microscopy of Normal Human and Keratoconus Cornea. Investig. Ophthalmol. Vis. Sci. 48, 1087-1094 (2007).

19. Meek, K. M. \& Boote, C. The use of X-ray scattering techniques to quantify the orientation and distribution of collagen in the corneal stroma. Prog. Retin. Eye Res. 28, 369-392 (2009).

20. Nguyen, T. M., Aubry, J. F., Fink, M., Bercoff, J. \& Tanter, M. In Vivo Evidence of Porcine Cornea Anisotropy Using Supersonic Shear Wave Imaging. Investig. Ophthalmol. Vis. Sci. 55, 7545-7552 (2014).

21. Elsheikh, A. et al. Experimental Assessment of Corneal Anisotropy. J. Refract. Surg. 24, 178-187 (2008).

22. Singh, M. et al. Investigating Elastic Anisotropy of the Porcine Cornea as a Function of Intraocular Pressure With Optical Coherence Elastography. J. Refract. Surg. 32, 562-567 (2016). 
23. Komai, Y. \& Ushiki, T. The three-dimensional organisation of collagen fibrils in the human cornea and sclera. Investig. Ophthalmol. Vis. Sci. 32, 2244-2258 (1991).

24. Meek, K. M. \& Quantock, A. J. The Use of X-ray Scattering Techniques to Determine Corneal Ultrastructure. Prog. Retin. Eye Res. 20, 95-137 (2001).

25. Meek, K. M. \& Leonard, D. W. Ultrastructure of the corneal stroma: a comparative study. Biophys. J. 64, 273-280 (1993).

26. Van Blokland, G. J. \& Verhelst, S. C. Corneal polarization in the living human eye explained with a biaxial model. J. Opt. Soc. Am. A 4, 82 (1987).

27. Shute, C. C. Haidinger's brushes and predominant orientation of collagen in corneal stroma. Nature 250, 163-164 (1974).

28. Donohue, D. J., Stoyanov, B. J., McCally, R. L. \& Farrell, R. A. Numerical modeling of the cornea's lamellar structure and birefringence properties. J. Opt. Soc. Am. A 12, 1425 (1995).

29. Thomsen, L. Weak elastic anisotropy. Geophysics 51, 1954-1966 (1986).

30. Dawson, D. G., Ubels, J. L. \& Edelhauser, H. F. Cornea and Sclera. In Adler's Physiology of the Eye, 71-130 (Elsevier Inc., 2011), eleventh edn.

31. Shao, P. et al. Spatially-resolved Brillouin spectroscopy reveals biomechanical abnormalities in mild to advanced keratoconus in vivo. Sci. Reports 9, 7467 (2019).

32. Fatt, I. \& Weissman, B. A. Cornea I: Form, Swelling Pressure, Transport Processes, and Optics. In Physiology of the Eye, chap. 6, 97-149 (Elsevier Inc., Amsterdam, Netherlands, 1992), 2nd edn.

33. Leonard, D. W. \& Meek, K. M. Refractive Indices of the Collagen Fibrils and Extrafibrillar Material of the Corneal Stroma. Biophys. J. 72, 1382-1387 (1997).

34. Hoffmeister, B. K., Verdonk, E. D., Wickline, S. A. \& Miller, J. G. Effect of collagen on the anisotropy of quasi-longitudinal mode ultrasonic velocity in fibrous soft tissues: A comparison of fixed tendon and fixed myocardium. J. Acoust. Soc. Am. 96, 1957-1964 (1994).

35. Kuo, P. L., Li, P. C. \& Li, M. L. Elastic properties of tendon measured by two different approaches. Ultrasound Medicine Biol. 27, 1275-1284 (2001).

36. Lim, D. J. Human tympanic membrane: An ultrastructural observation. Acta Oto-Laryngologica 70, 176-186 (1970).

37. Williams, K. R., Blayney, A. W. \& Lesser, T. H. Mode shapes of a damaged and repaired tympanic membrane as analysed by the finite element method. Clin. Otolaryngol. Allied Sci. 22, 126-131 (1997).

38. Schacht, S. A. L., Stahn, P., Hinsberger, M. \& Schick, B. Laser-induced tissue remodeling within the tympanic membrane. J. Biomed. Opt. 23, 1 (2018).

39. Yao, W. et al. Collagen fiber orientation and dispersion in the upper cervix of non-pregnant and pregnant women. PLoS ONE 11, 1-20 (2016). 
40. Shi, L. et al. Anisotropic Material Characterization of Human Cervix Tissue Based on Indentation and Inverse Finite Element Analysis. J. Biomech. Eng. 141, 1-13 (2019).

41. O'Connell, M. K. et al. The Three-Dimensional Micro- and Nanostructure of the Aortic Medial Lamellar Unit Measured Using 3D Confocal and Electron Microscopy Imaging. Matrix Biol. 27, 171-181 (2008).

42. Gasser, T. C. et al. Spatial orientation of collagen fibers in the abdominal aortic aneurysm's wall and its relation to wall mechanics. Acta Biomater. 8, 3091-3103 (2012).

43. Courtney, T., Sacks, M. S., Stankus, J., Guan, J. \& Wagner, W. R. Design and analysis of tissue engineering scaffolds that mimic soft tissue mechanical anisotropy. Biomaterials 27, 3631-3638 (2006).

44. de Mulder, E. L., Buma, P. \& Hannink, G. Anisotropic Porous Biodegradable Scaffolds for Musculoskeletal Tissue Engineering. Materials 2, 1674-1696 (2009).

45. Schwan, J. et al. Anisotropic engineered heart tissue made from laser-cut decellularized myocardium. Sci. Reports 6, 1-12 (2016).

46. Ramier, A. et al. In vivo measurement of shear modulus of the human cornea using optical coherence elastography. Sci. Reports 10 (2020).

47. Pitre, J. J. et al. Nearly-incompressible transverse isotropy (NITI) of cornea elasticity: model and experiments with acoustic micro-tapping OCE. Sci. Reports 10, 1-14 (2020).

48. Kirby, M. A. et al. Delineating Corneal Elastic Anisotropy in a Porcine Model Using Noncontact OCT Elastography and Ex Vivo Mechanical Tests. Ophthalmol. Sci. 1, 100058 (2021).

49. Scarcelli, G. et al. Noncontact three-dimensional mapping of intracellular hydromechanical properties by Brillouin microscopy. Nat. Methods 12, 1132-1134 (2015).

\section{Acknowledgements}

The authors thank Dr. Theo Seiler and Dr. Doyle Stulting for obtaining the human data and Neema Kumar and Jie Zhao for assistance with sample preparation and TEM imaging. This study was supported by funding from the National Institutes of Health, grants R01-EY025454, P41-EB015903, R01-EB027653, and R41-EY028820.

\section{Author contributions statement}

A.M.E., P.S., and S.H.Y. designed the experiments. A.M.E. conducted the experiments and analyzed the data. A.M.E. and S.H.Y. wrote the manuscript. 


\section{Additional information}

Competing interests A.M.E., P.S. and S.H.Y. hold issued and pending patents related to the technology. S.H.Y. is the scientific founder of Intelon Optics, Inc., which licensed the patents. 
a

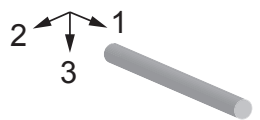

b

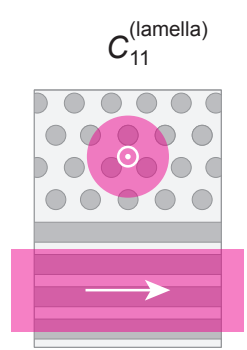

Fibrils $(f)$

Extrafibrillar matrix $(m)$

C

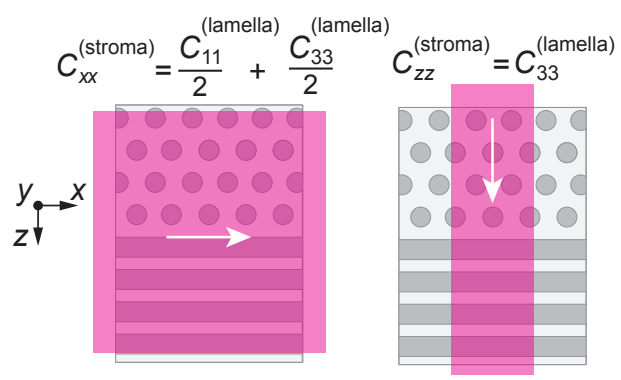

Figure 1. Brillouin measurements at different laser incidence angles detect tissue anisotropy. a, Within each lamella, collagen fibers $(f)$ are co-aligned within a gel matrix $(m)$. We define a coordinate system $(1,2,3)$ for each lamella in which the 1-direction is always aligned with the fibril axis. $\mathbf{b}$, The elastic modulus in the 1-direction $C_{11}$ is different than the moduli orthogonal to the fibril axis, $C_{22}$ and $C_{33}$. By reducing the laser spot size, it is possible to resolve individual lamellae using Brillouin microscopy. When the laser spot size covers multiple lamellae, we measure a combined modulus. For the bulk stroma we define the $z$-direction as orthogonal to the cornea and the $x-y$ plane as tangential. c, Cut-away view through the stromal depth showing stacked lamellae. The cornea is mechanically anisotropic, meaning that the longitudinal moduli $\left(C_{i i}\right)$ have directional dependence. This can be probed using Brillouin microscopy with varying laser incidence angle $\theta$.

a

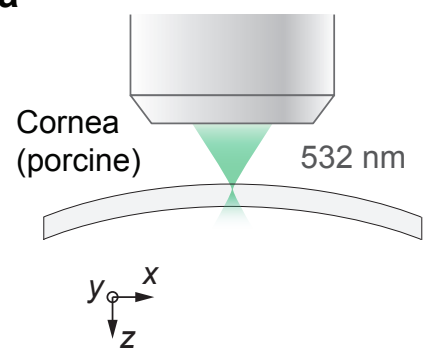

b

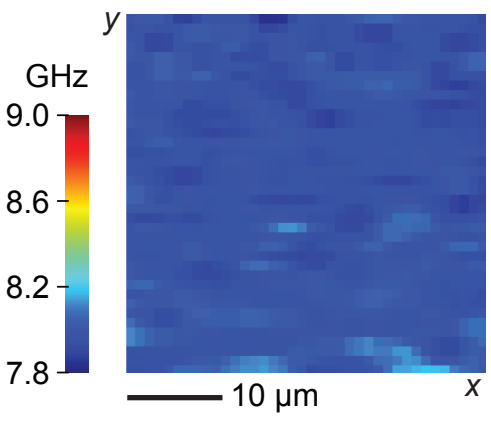

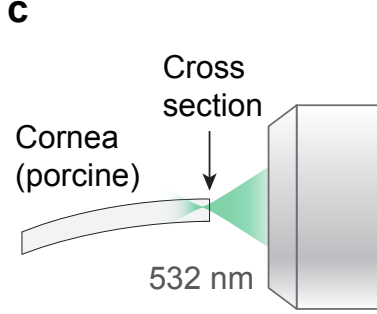

d

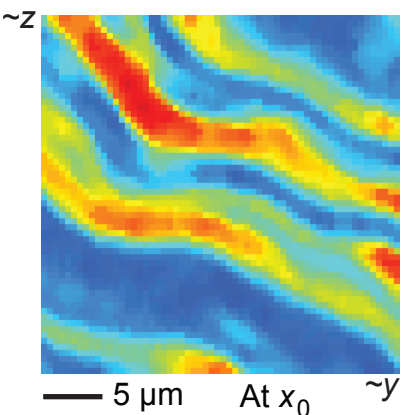

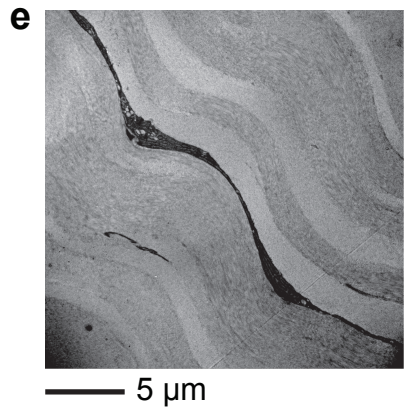
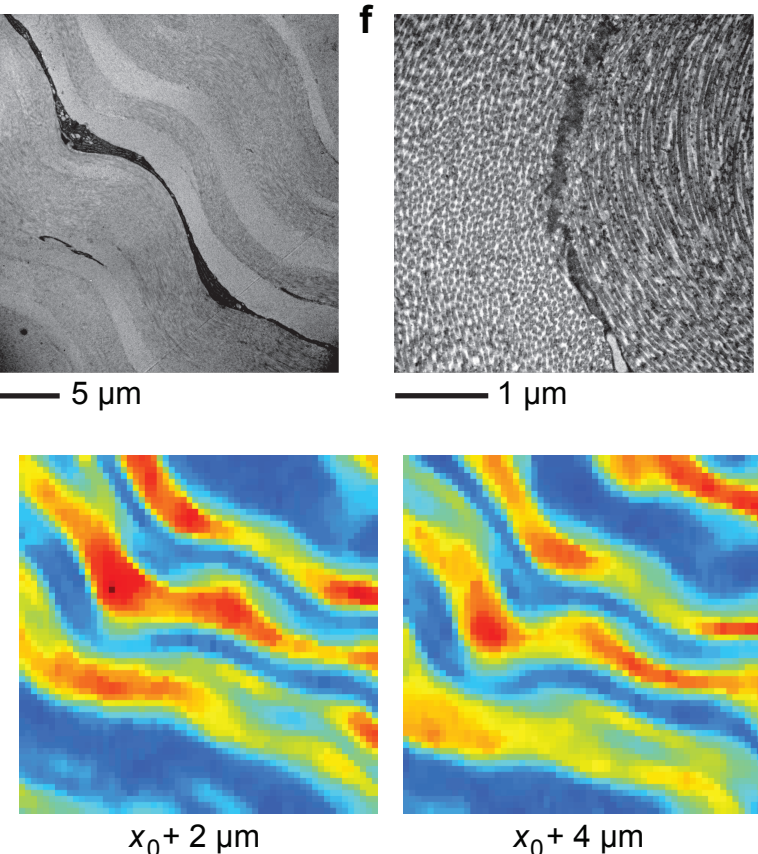

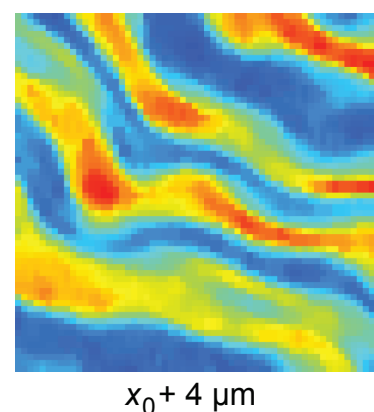

Figure 2. High-resolution Brillouin imaging of ex vivo porcine cornea with a $532 \mathrm{~nm}$ wavelength. a, Schematic showing en face Brillouin imaging of the cornea using a $532 \mathrm{~nm}$ laser focused to a spot size of approximately $0.5 \mu \mathrm{m}$. b. Brillouin image of ex vivo porcine cornea measured en-face, showing relatively uniform Brillouin value across the $x-y$ plane. c, Schematic showing Brillouin imaging of the corneal cross-section. The laser was focused just below the surface of the cross-section. $\mathbf{d}$, Brillouin images of corneal cross-section at three different (relative) depths, revealing a lamellar pattern. e Transmission electron microscopy (TEM) image of corneal cross-section showing individual lamellae with alternating fibril orientations. A keratocyte is visible in between the two lamellae at the center of the image (keratocyte appears black with substructure). $\mathbf{f}$, Higher resolution TEM image of the same region. Individual collagen fibrils can be seen aligned to the preferred directions in two adjacent lamellae. 
a
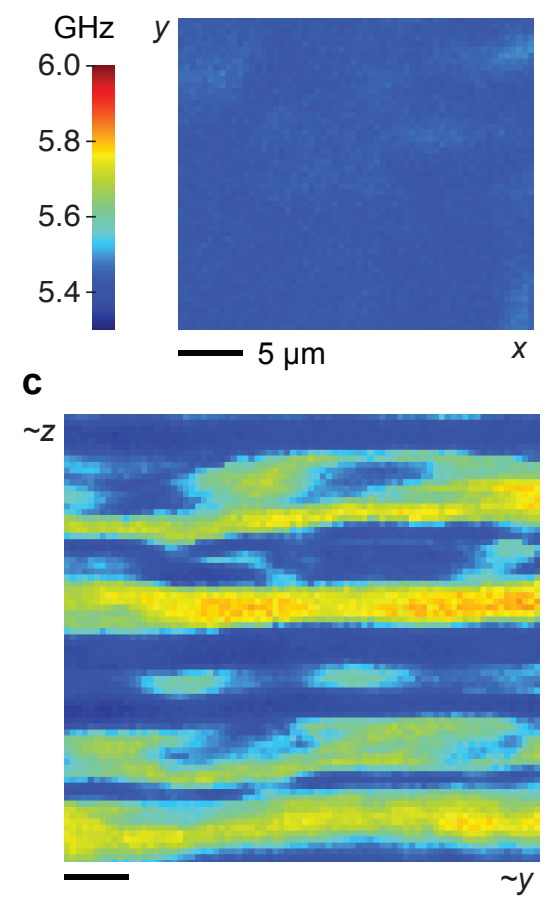

f

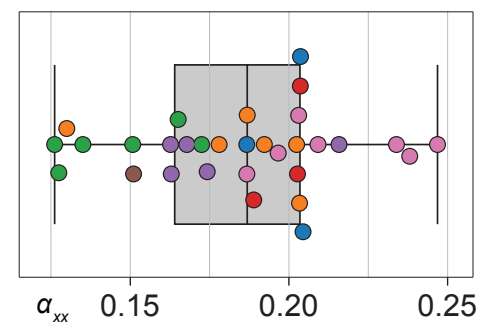

b
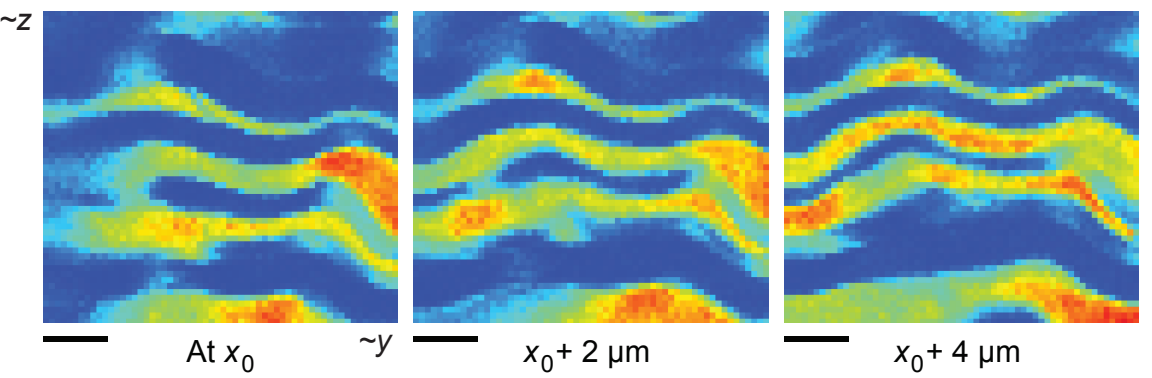

d
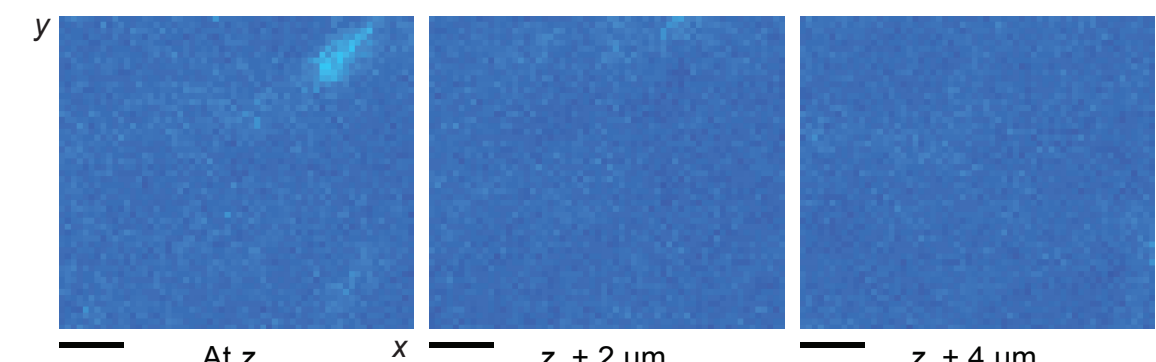

e
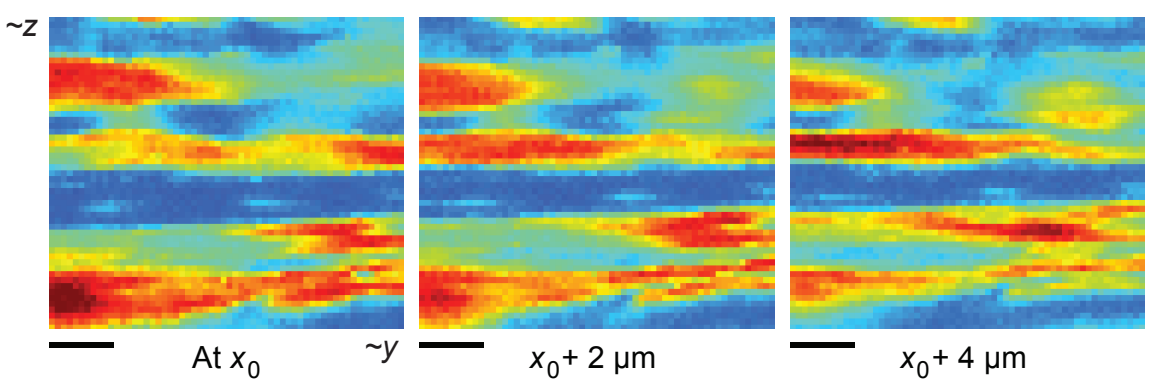

Figure 3. High-resolution Brillouin imaging of ex vivo porcine corneas with a $780 \mathrm{~nm}$ wavelength. a, En-face image showing relatively uniform Brillouin value across the $x-y$ plane. Note that the colormap scale used in this figure is different from that of Fig. $2 b$ (due to the wavelength difference). b. Brillouin images of the same corneal sample measured in cross-section at three different (relative) depths, revealing a lamellar pattern. $\mathbf{c}$ Larger Brillouin image of a second corneal sample measured in cross-section. d, Brillouin images of a third corneal sample measured en-face at three different (relative) depths. e, Brillouin images of the same (third) corneal sample measured in cross-section at three different (relative) depths. f Distribution of $\alpha_{x x}$ values estimated from 31 cross-sectional Brillouin images of 7 ex vivo corneas from 7 pigs. Points displayed in the same color represent data taken at different spatial locations in the same corneal cross-section.

Box-plot: center line, median; box limits, upper and lower quartiles; whiskers, range of data. 
a
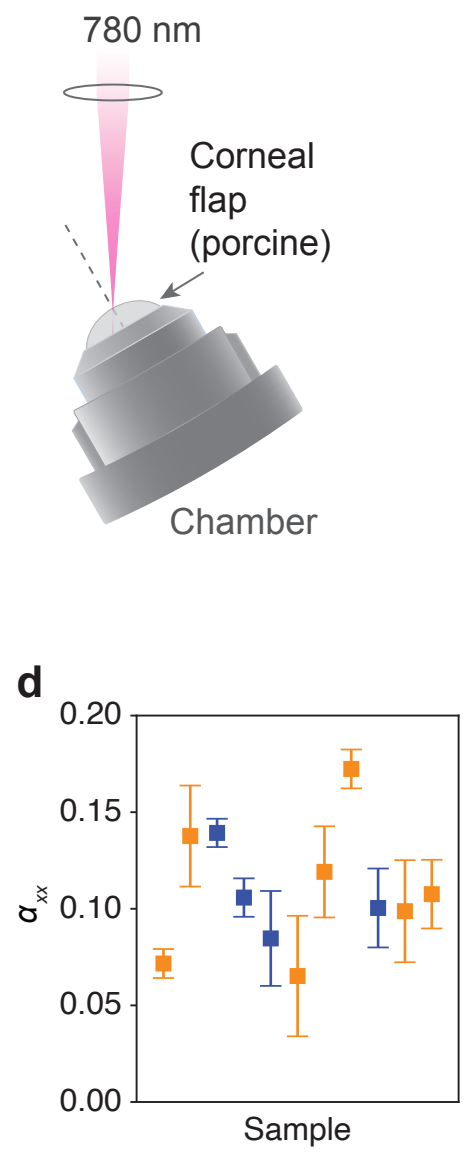

b
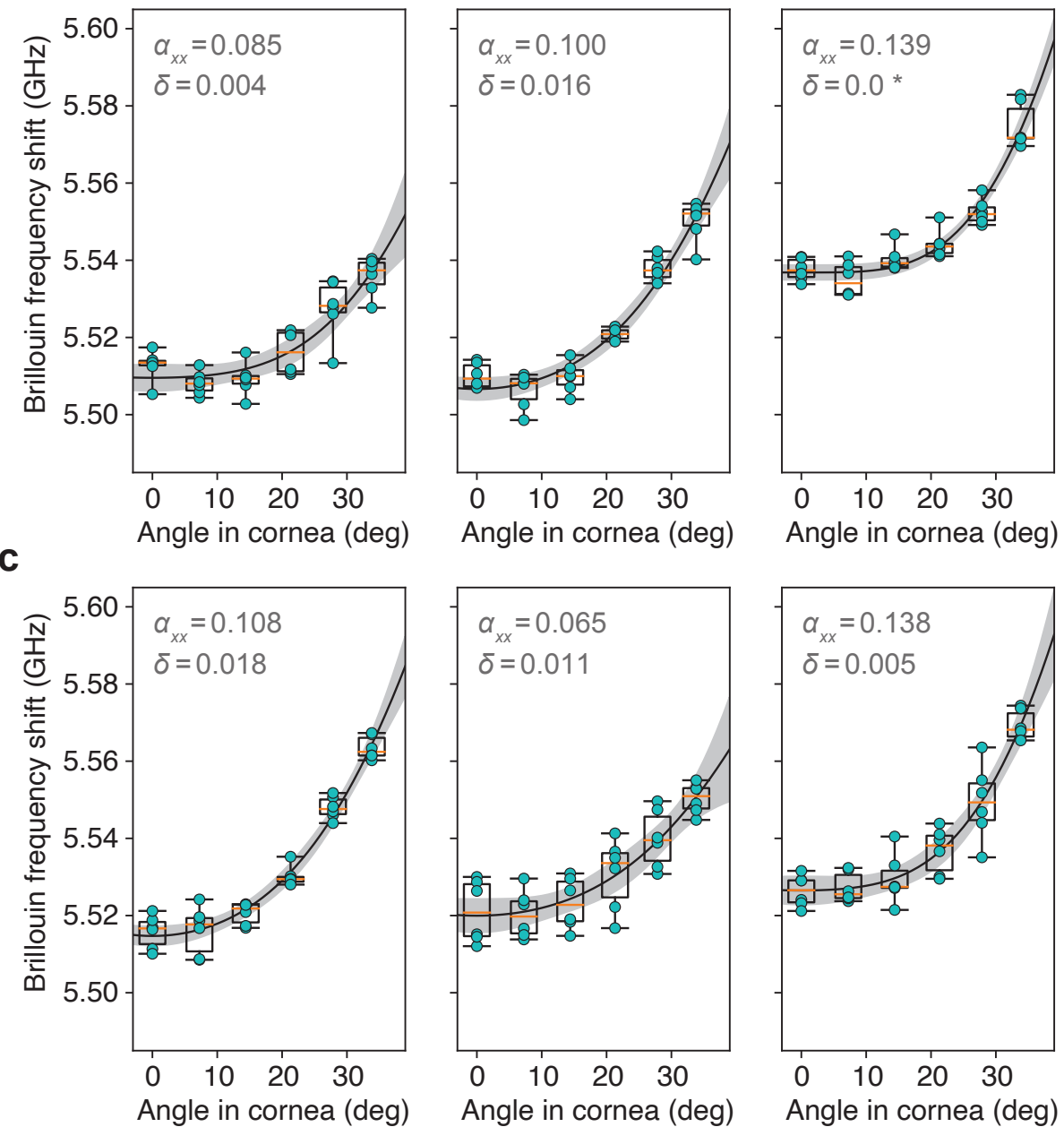

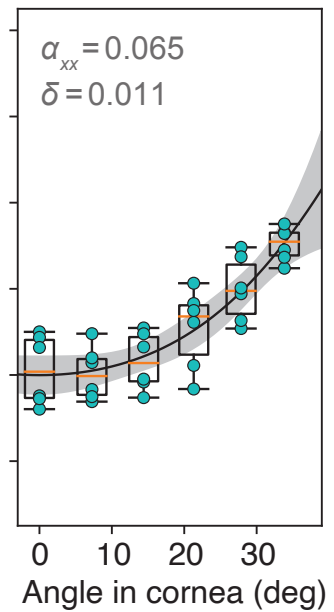

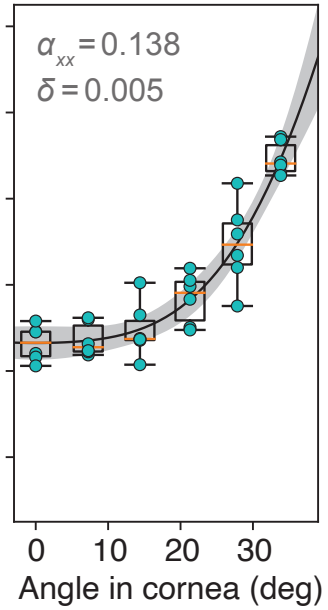

Figure 4. Measurement of Brillouin angle-dependence in ex vivo porcine cornea. a, Schematic showing Brillouin angle-dependence measurements of the cornea mounted in a Barron chamber using a 0.1-NA, $780 \mathrm{~nm}$ optical beam. b, Plots of Brillouin frequency shift measured at the center of the cornea with different in-tissue angles for 3 porcine samples (different animals). For each sample, the cycle of angle measurements was repeated six times (alternating increasing and decreasing angle) to minimize the impact of environmental drifts. Individual measurement points are shown in cyan, along with box plots for all 6 points at each angle setting. Best-fit curves are plotted (solid line) with 95\% confidence bands (grey). Fitted values of $\alpha_{x x}$ and $\delta$ are shown.

c, Plots of Brillouin frequency shift measured $2 \mathrm{~mm}, 3 \mathrm{~mm}$, and $4 \mathrm{~mm}$ away from center (left to right, respectively) with different in-tissue angles for 3 porcine samples (different animals). d, Distribution of fitted $\alpha_{x x}^{\text {(stroma) }}$ values for 11 corneas from 11 different animals. Error bars indicate confidence ranges. Points in blue correspond to the central cornea, while points in orange correspond to other locations $2-4 \mathrm{~mm}$ off center.

Box-plots: center line, median; box limits, upper and lower quartiles; whiskers, range of data. 


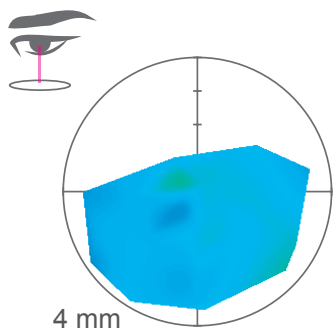

C

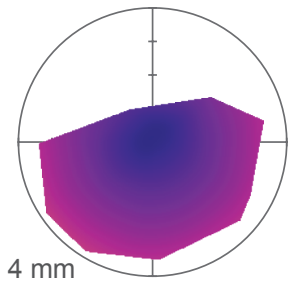

e

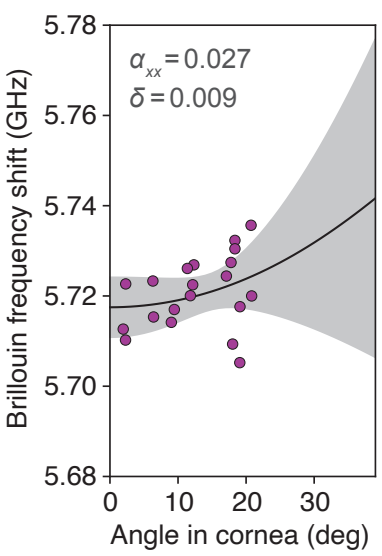

b
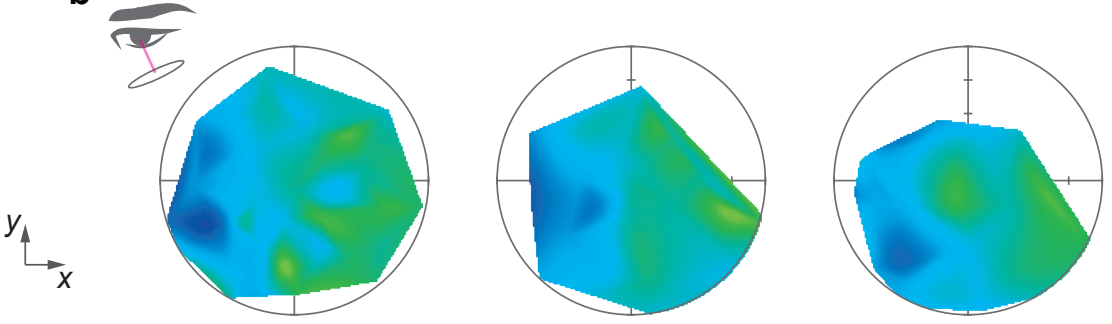

$\mathrm{GHz}$

$-5.80$

5.75

$-5.70$

5.65

5.60

d
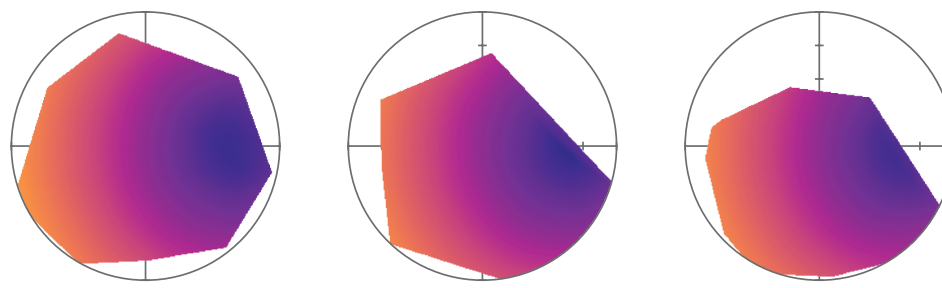

deg

$-40$

$-30$

$-20$

10

f

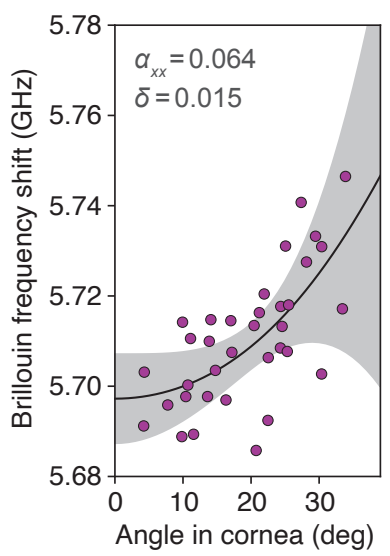

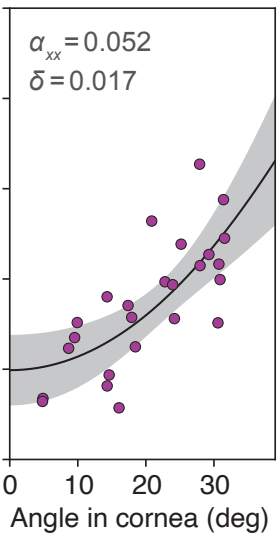

Figure 5. Anisotropy in vivo in Brillouin maps of the human cornea $(n=4,3: 1 \mathrm{M}: \mathrm{F}, 31.5 \pm 2.4 \mathrm{Y} / \mathrm{O})$. a, Brillouin frequency shift map of a normal subject with laser incidence angle close to $0^{\circ}$ at the corneal apex (see diagram at left). $\mathbf{b}$, Brillouin maps of three normal subjects for a steeper set of laser incidence angles (see diagram). c-d, Maps showing computed laser incidence angle corresponding to the Brillouin maps above. The laser-cornea incidence angle varies slightly as the laser is translated in $\mathrm{x}$ (left-right) and y (up-down) to create the Brillouin map due to corneal curvature. e-f, Plot of Brillouin values for each map versus computed incidence angle. The increasing Brillouin shift with angle is consistent with expected corneal anisotropy. Individual map data points (shown in magenta) were fit to the weak transverse isotropy model (Eq. 3). Best-fit curves are plotted (solid line) with 95\% confidence bands (grey). Fitted values of $\alpha_{x x}$ and $\delta$ are shown. 


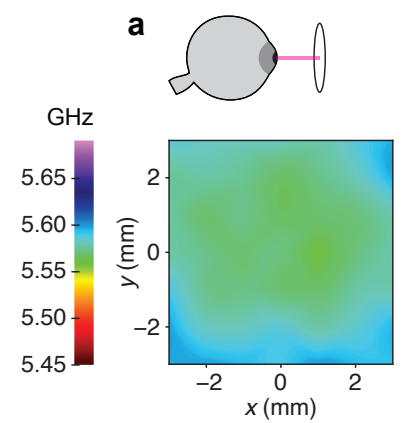

b
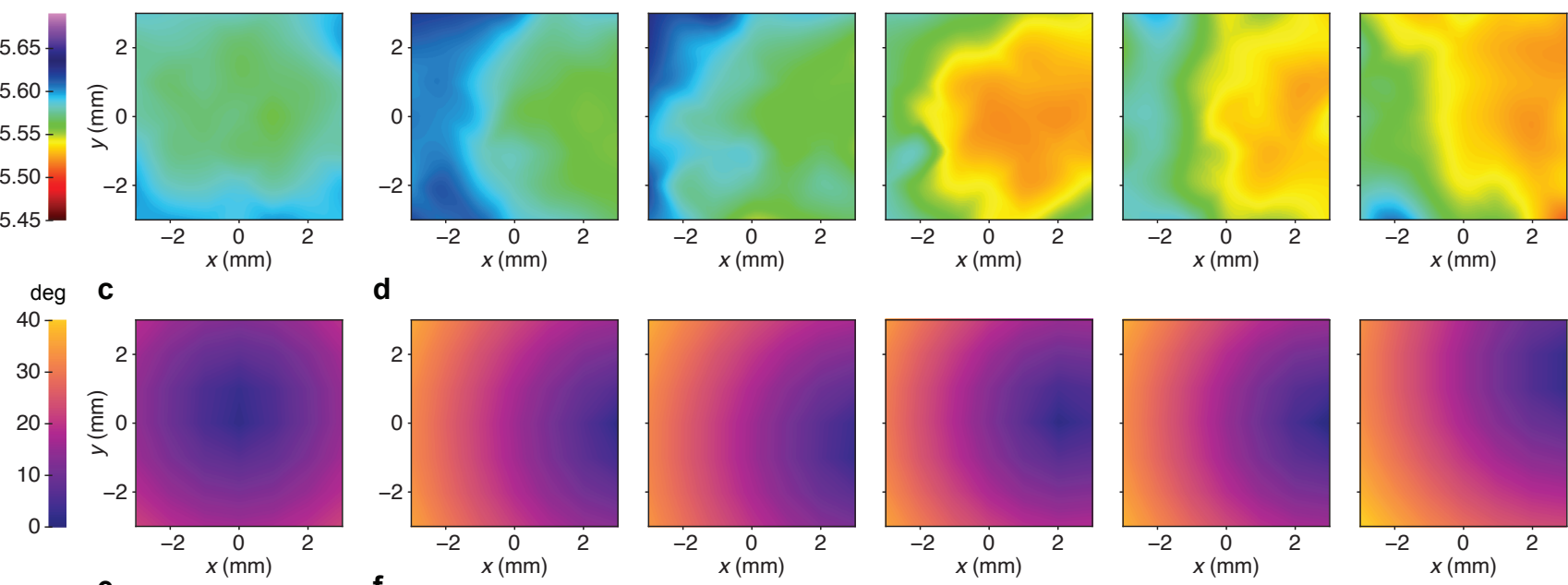

d
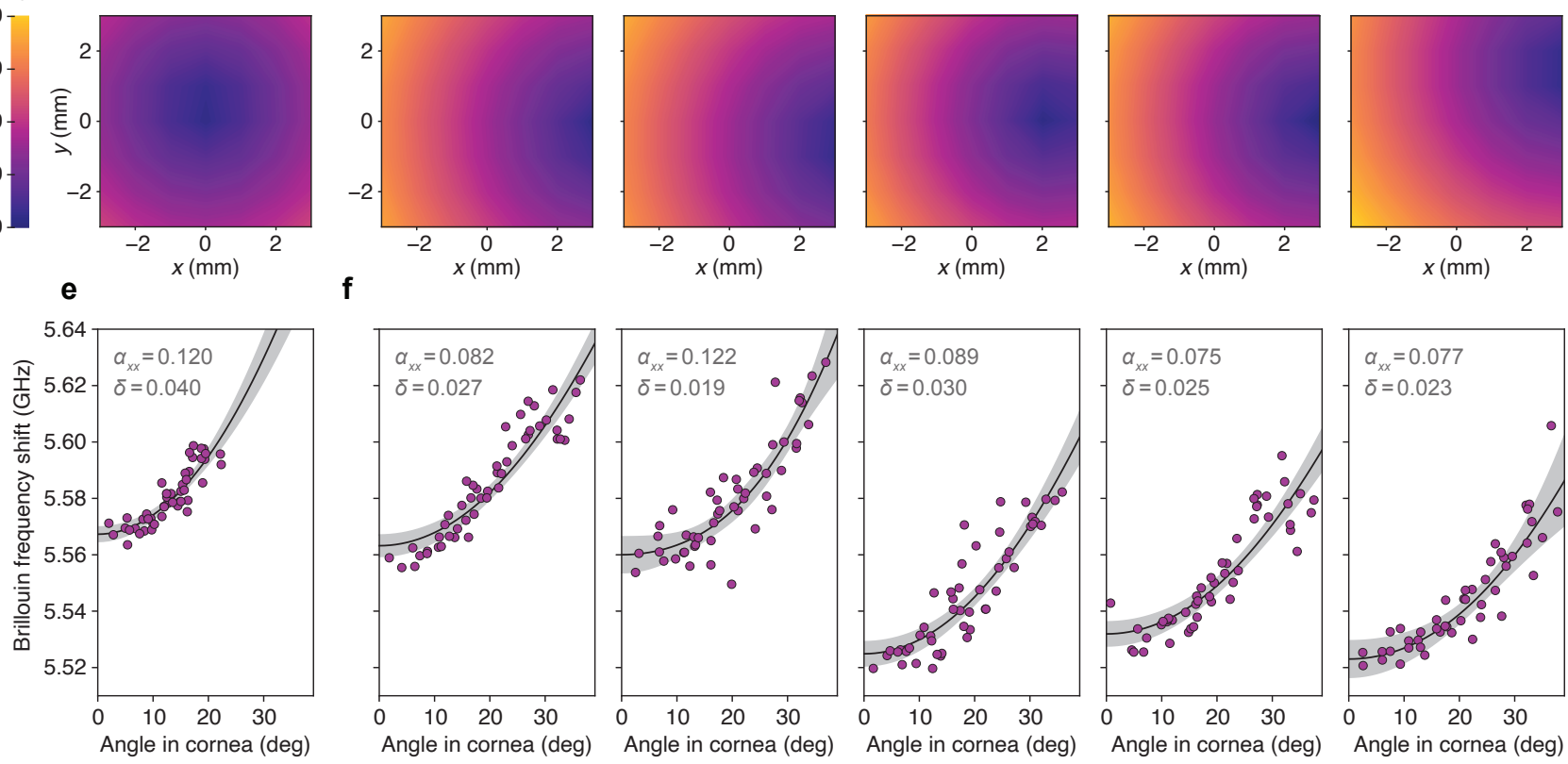

Figure 6. Anisotropy in Brillouin maps of ex vivo porcine cornea. a, Brillouin frequency shift map of intact porcine cornea with laser incidence angle close to $0^{\circ}$ at the corneal apex (see diagram above). b, Brillouin map of the same cornea (left) and the corneas of four other animals (right) for a steeper set of laser incidence angles (see diagram above). c-d, Maps showing computed laser incidence angle corresponding to the Brillouin maps above. The laser-cornea incidence angle varies slightly as the laser is translated in $\mathrm{x}$ (left-right) and y (up-down) to create the Brillouin map due to corneal curvature. e-f, Plot of Brillouin values for each map versus computed incidence angle. The increasing Brillouin shift with angle is consistent with expected corneal anisotropy. Individual map data points (shown in magenta) were fit to the weak transverse isotropy model (Eq. 3). Best-fit curves are plotted (solid line) with 95\% confidence bands (grey). Fitted values of $\alpha_{x x}$ and $\delta$ are shown. 


\title{
Supplementary Materials for Measuring mechanical anisotropy of the cornea with Brillouin microscopy
}

\author{
Amira M. Eltony ${ }^{1}$, Peng Shao ${ }^{1,+}$, and Seok-Hyun Yun ${ }^{1,2, *}$ \\ ${ }^{1}$ Harvard Medical School and Wellman Center for Photomedicine, Massachusetts General Hospital, Boston, MA, \\ 02114, USA \\ ${ }^{2}$ Harvard-MIT Health Sciences and Technology, Cambridge, MA, 02139, USA \\ +Present affiliation: Reveal Surgical Inc., Montréal, QC, H2N 1A4, Canada \\ *syun@hms.harvard.edu
}

\section{Composite model of a corneal lamella}

Let $\sigma_{i}^{(k)}$ and $s_{j}^{(k)}$ denote the $i$-th stress and $j$-th strain elements, respectively, in Voigt notation. Here, '1' is for ' $x x$ ', '2' for ' $y y$ ', '3' for ' $z z$ ', '4' for ' $y z$ ', '5' for ' $x z$ ', and '6' for ' $x y$ ' for Cartesian coordinates used to describe the cornea (Fig. 1C). On the other hand, '1' is for '11', '2' for '22', '3' for '33', '4' for '23', '5' for '13', and '6' for '12' for the 123-coordinate system defined with respect to a single collagen fibril (Fig. 1A). $C_{i j}^{(k)}$ denotes the $i j$-th elastic modulus for material ' $k$ ', such that we have stress-strain relations of the form (Voigt notation):

$$
\left[\begin{array}{c}
\sigma_{1}^{(k)} \\
\sigma_{2}^{(k)} \\
\sigma_{3}^{(k)} \\
\sigma_{4}^{(k)} \\
\sigma_{5}^{(k)} \\
\sigma_{6}^{(k)}
\end{array}\right]=\left[\begin{array}{cccccc}
C_{11}^{(k)} & C_{12}^{(k)} & C_{13}^{(k)} & 0 & 0 & 0 \\
C_{12}^{(k)} & C_{22}^{(k)} & C_{23}^{(k)} & 0 & 0 & 0 \\
C_{13}^{(k)} & C_{23}^{(k)} & C_{33}^{(k)} & 0 & 0 & 0 \\
0 & 0 & 0 & C_{44}^{(k)} & 0 & 0 \\
0 & 0 & 0 & 0 & C_{55}^{(k)} & 0 \\
0 & 0 & 0 & 0 & 0 & C_{66}^{(k)}
\end{array}\right]\left[\begin{array}{c}
s_{1}^{(k)} \\
s_{2}^{(k)} \\
s_{3}^{(k)} \\
s_{4}^{(k)} \\
s_{5}^{(k)} \\
s_{6}^{(k)}
\end{array}\right]
$$

We define a coordinate system for the lamella in which the 1-direction is parallel to the fibril axis, and the 2- and 3-directions are orthogonal to it (Fig. 1). For Brillouin measurements at a $180^{\circ}$ angle, light scatters from longitudinal elastic waves which have displacement only along the direction of wave propagation. For example, an elastic wave propagating in the 1-direction has $s_{2}^{(k)}=s_{3}^{(k)}=0$ and hence $\sigma_{1}^{(k)}=C_{11}^{(k)} s_{1}^{(k)}$.

First, consider the case of loading parallel to the fibril axis (1-direction). The stress in the composite is the sum of the stresses in all of the fibrils $\left(\sigma_{1}^{(f)}\right)$ and in the extrafibrillar matrix $\left(\sigma_{1}^{(m)}\right.$ ), weighted by their volume fractions (law of mixtures):

$$
\sigma_{1}^{(\text {lamella })}=\sigma_{1}^{(f)} V^{(f)}+\sigma_{1}^{(m)}\left(1-V^{(f)}\right)
$$

If we assume that there is no slippage between the fibrils and the matrix, the strain of the fibers and the matrix must be equal 
(the 'isostrain rule'):

$$
s_{1}^{(\text {lamella) }}=s_{1}^{(f)}=s_{1}^{(m)}
$$

Hence, the longitudinal modulus $C_{11}^{(\text {lamella })}$ is given by:

$$
C_{11}^{(\text {lamella) }}=C_{11}^{(f)} V^{(f)}+C_{11}^{(m)}\left(1-V^{(f)}\right)
$$

Likewise, in the case of loading perpendicular to the fiber axis, the fibrils and the matrix experience different strains, but equal stress ( 'isostress rule'):

$$
\begin{aligned}
& \frac{1}{C_{22}^{\text {(lamella })}}=\frac{V^{(f)}}{C_{22}^{(f)}}+\frac{\left(1-V^{(f)}\right)}{C_{22}^{(m)}} \\
& \frac{1}{C_{33}^{(\text {lamella })}}=\frac{V^{(f)}}{C_{33}^{(f)}}+\frac{\left(1-V^{(f)}\right)}{C_{33}^{(m)}}
\end{aligned}
$$

If we assume that the extrafibrillar matrix is isotropic, $C_{11}^{(m)}=C_{33}^{(m)}$, and that the fibers are radially symmetric, $C_{22}^{(f)}=C_{33}^{(f)}$, we obtain:

$$
\begin{aligned}
& C_{11}^{(\text {lamella })}=C_{11}^{(f)} V^{(f)}+C_{11}^{(m)}\left(1-V^{(f)}\right) \\
& C_{22}^{(\text {lamella })}=C_{33}^{(\text {lamella })}=\frac{C_{11}^{(m)} V^{(f)}+C_{33}^{(f)}\left(1-V^{(f)}\right)}{C_{11}^{(m)} C_{33}^{(f)}}
\end{aligned}
$$

So we see that an individual lamella is transverse isotropic with plane of symmetry $2-3$ (i.e. orthogonal to the fibril axis).

\section{Model of an orthogonal stack of lamellae}

To compute the properties of the bulk stroma, we sum over the contributions of all the lamellae. The axes of the collagen fibrils in successive lamellae typically lie along orthogonal meridians in the medial-lateral and superior-inferior directions [Meek et al. Prog. Retin. Eye Res. 20, 95-137 (2001)]. Therefore, we model the stroma as a summation of layers with half oriented in the medial-lateral direction and half in the superior-inferior direction. For the stroma, we define a coordinate system $(x, y, z)$ with the $z$-direction orthogonal to the cornea, and the $x$ - and $y$-directions tangential (medial-lateral and superior-inferior). In the tangential plane $x-y$, the 'isostrain rule' applies similarly to Eq. $\mathrm{S} 4$, so by symmetry:

$$
C_{x x}^{(\text {stroma })}=C_{y y}^{(\text {stroma })}=\frac{1}{2} C_{11}^{\text {(lamella) }}+\frac{1}{2} C_{33}^{\text {(lamella) }}
$$


The $z$-direction is orthogonal to the fiber axes in all lamellae, so:

$$
C_{z z}^{(\text {stroma })}=C_{22}^{(\text {lamella })}=C_{33}^{(\text {lamella })}
$$

In this model, the cornea is transverse isotropic with plane of symmetry $x-y$.

The stress-strain relation of a transverse-isotropic material in the $x y z$ coordinate system can be written as:

$$
\left[\begin{array}{c}
\sigma_{x x} \\
\sigma_{y y} \\
\sigma_{z z} \\
\sigma_{y z} \\
\sigma_{y z} \\
\sigma_{x y}
\end{array}\right]=\left[\begin{array}{cccccc}
C_{x x} & C_{x y} & C_{x z} & 0 & 0 & 0 \\
C_{x y} & C_{x x} & C_{x z} & 0 & 0 & 0 \\
C_{x z} & C_{x z} & C_{z z} & 0 & 0 & 0 \\
0 & 0 & 0 & G_{y z} & 0 & 0 \\
0 & 0 & 0 & 0 & G_{y z} & 0 \\
0 & 0 & 0 & 0 & 0 & G_{x y}
\end{array}\right]\left[\begin{array}{c}
s_{x x} \\
s_{y y} \\
s_{z z} \\
s_{y z} \\
s_{x z} \\
s_{x y}
\end{array}\right]
$$

Here, $G_{y z}\left(=G_{x z}\right)$ and $G_{x y}$ correspond to shear moduli in the yz- (xz-) and xy-plane, respectively. By symmetry, $C_{x x}-C_{x y}=2 G_{x y}$, and there are five independent parameters in the stiffness matrix.

\section{Direction-dependent longitudinal modulus}

For transverse isotropic materials, there is an analytic expression for the effective longitudinal modulus at an angle $\theta$ to the $z$-axis (assuming material plane of symmetry $x-y$ ):

$$
C(\theta)=\frac{1}{2}\left[C_{x x} \sin ^{2}(\theta)+C_{z z} \cos ^{2}(\theta)+G_{y z}+D(\theta)\right]
$$

with $D(\theta)=\sqrt{\left[\left(C_{x x}-G_{y z}\right) \sin ^{2}(\theta)-\left(C_{z z}-G_{y z}\right) \cos ^{2}(\theta)\right]^{2}+\left(C_{x z}+G_{y z}\right)^{2} \sin ^{2}(2 \theta)}$ and mass density $\rho$. When $\theta=0, C(0)=$ $C_{z z}$, and at $\theta=\pi / 2, C(\pi / 2)=C_{x x}$. This range of possible $C(\theta)$ values characterizes the scale of the anisotropy of the tissue. We introduce the parameters $\alpha_{x x}, \alpha_{x z}$, and $\alpha_{y z}$, defined as:

$$
\begin{aligned}
& \alpha_{x x}=\frac{C_{x x}}{C_{z z}}-1 \\
& \alpha_{x z}=\frac{C_{x z}}{C_{z z}}-1 \\
& \alpha_{y z}=\frac{G_{y z}}{C_{z z}}
\end{aligned}
$$


This allows us to rewrite the expression for the longitudinal modulus as follows:

$$
\begin{aligned}
C(\theta)=\frac{C_{z z}}{2}\left[\left(1+\alpha_{x x}\right) \sin ^{2}(\theta)\right. & \left.+\cos ^{2}(\theta)+\alpha_{y z}\right] \\
+ & \frac{C_{z z}}{2} \sqrt{\left[\left(1+\alpha_{x x}-\alpha_{y z}\right) \sin ^{2}(\theta)-\left(1-\alpha_{y z}\right) \cos ^{2}(\theta)\right]^{2}+\left(1+\alpha_{x z}+\alpha_{y z}\right)^{2} \sin ^{2}(2 \theta)}
\end{aligned}
$$

For soft tissues like the cornea, the shear modulus $G_{y z}$ is typically much smaller than the longitudinal moduli $C_{z z}$, so $\alpha_{y z} \ll 1$. Assuming that the anisotropy of the cornea is also relatively small, $\alpha_{x x}, \alpha_{x z} \ll 1$. We can expand Eq. S12 to first order in the small parameters $\alpha_{x x}, \alpha_{x z}$, and $\alpha_{y z}$, which yields:

$$
C(\theta) \approx C(0)\left[1+\left(2 \alpha_{x z}+4 \alpha_{y z}\right) \sin ^{2}(\theta) \cos ^{2}(\theta)+\alpha_{x x} \sin ^{4}(\theta)\right]
$$

where $C(0)=C_{z z}$ as before.

\section{The anisotropic parameter $\alpha_{x x}$}

The small parameter $\alpha_{x x}$ (defined in Eq. S11a) characterizes the degree of anisotropy of a transverse isotropic material. For a single lamella, this anisotropic parameter, $\alpha_{11}^{\text {(lamella) }}$, is given by:

$$
\begin{aligned}
\alpha_{11}^{(\text {lamella })} & =\frac{C_{11}^{\text {(lamella) }}}{C_{33}^{\text {(lamella) }}}-1 \\
& =\frac{\left(C_{11}^{(f)} V^{(f)}+C_{11}^{(m)}\left(1-V^{(f)}\right)\right)\left(C_{11}^{(m)} V^{(f)}+C_{33}^{(f)}\left(1-V^{(f)}\right)\right)}{C_{33}^{(f)} C_{11}^{(m)}}-1
\end{aligned}
$$

If we set $C_{11}^{(m)}=\beta_{1} C_{33}^{(f)}$ and $C_{11}^{(f)}=\beta_{2} C_{33}^{(f)}$, we obtain:

$$
\alpha_{11}^{\text {(lamella) }}=\frac{\left(\beta_{2} V^{(f)}+\beta_{1}\left(1-V^{(f)}\right)\right)\left(\beta_{1} V^{(f)}+1-V^{(f)}\right)}{\beta_{1}}-1
$$

Using Eqs. (S7) and (S8), the anisotropic parameter $\alpha_{x x}$ for an orthogonal stack of lamellae is related to the anisotropy of each lamella:

$$
\begin{aligned}
\alpha_{x x}^{\text {(stroma) }} & =\frac{C_{x x}^{\text {(stroma) }}}{C_{z z}^{\text {(stroma) }}}-1=\frac{C_{11}^{\text {(lamella) }}+C_{33}^{\text {(lamella })}}{2 C_{33}^{\text {(lamella) }}}-1 \\
& =\frac{1}{2} \alpha_{11}^{\text {(lamella) }}
\end{aligned}
$$




\section{Tables of fit parameters for all porcine samples}

\begin{tabular}{|c|c|c|c|c|}
\hline$\Omega(0)$ & $\alpha_{x x}^{\text {(stroma) }}$ & $\delta$ & $R^{2}$ & Measurement location \\
\hline $5.537 \pm 0.001$ & $0.139 \pm 0.007$ & $0^{*}$ & 0.92 & center \\
$5.511 \pm 0.001$ & $0.106 \pm 0.010$ & $0^{*}$ & 0.78 & center \\
$5.510 \pm 0.002$ & $0.085 \pm 0.025$ & $0.004 \pm 0.007$ & 0.78 & center \\
$5.507 \pm 0.001$ & $0.100 \pm 0.020$ & $0.016 \pm 0.008$ & 0.93 & center \\
$5.524 \pm 0.003$ & $0.072 \pm 0.007$ & $0.024^{*}$ & 0.74 & $2 \mathrm{~mm}$ from center \\
$5.515 \pm 0.001$ & $0.108 \pm 0.018$ & $0.018 \pm 0.007$ & 0.96 & $2 \mathrm{~mm}$ from center \\
$5.520 \pm 0.002$ & $0.065 \pm 0.031$ & $0.011 \pm 0.012$ & 0.74 & $3 \mathrm{~mm}$ from center \\
$5.501 \pm 0.002$ & $0.119 \pm 0.024$ & $0.015 \pm 0.008$ & 0.93 & $3 \mathrm{~mm}$ from center \\
$5.496 \pm 0.001$ & $0.172 \pm 0.010$ & $0^{*}$ & 0.90 & $3 \mathrm{~mm}$ from center \\
$5.537 \pm 0.002$ & $0.099 \pm 0.026$ & $0.017 \pm 0.010$ & 0.90 & $3 \mathrm{~mm}$ from center \\
$5.527 \pm 0.002$ & $0.138 \pm 0.026$ & $0.005 \pm 0.007$ & 0.89 & $4 \mathrm{~mm}$ from center \\
\hline
\end{tabular}

Table S1. Angle-dependence of porcine corneas ex vivo. Please refer to Fig. 4. Fitted value \pm confidence interval for each parameter, coefficient of determination $\left(R^{2}\right)$, and measurement location for the 11 ex vivo porcine corneas measured. The $\delta$ parameter was constrained to $0<\delta<0.25 \alpha_{x x}^{\text {(stroma) }}$ * indicates $\delta$ at bound.

\begin{tabular}{|c|c|c|c|c|c|}
\hline Corneal ROC & $\Omega(0)$ & $\alpha_{x x}^{\text {(stroma) }}$ & $\delta$ & $R^{2}$ & Sample \\
\hline 8.72 & $5.567 \pm 0.001$ & $0.120 \pm 0.010$ & $0.040^{*}$ & 0.78 & 1 ( normal) \\
8.64 & $5.563 \pm 0.002$ & $0.082 \pm 0.005$ & $0.027^{*}$ & 0.84 & 1 (tilted) \\
8.79 & $5.560 \pm 0.003$ & $0.122 \pm 0.036$ & $0.019 \pm 0.014$ & 0.81 & 2 (tilted) \\
7.61 & $5.525 \pm 0.002$ & $0.089 \pm 0.007$ & $0.030^{*}$ & 0.79 & 3 (tilted) \\
7.89 & $5.532 \pm 0.002$ & $0.075 \pm 0.006$ & $0.025^{*}$ & 0.79 & 4 (tilted) \\
8.34 & $5.525 \pm 0.003$ & $0.115 \pm 0.032$ & $0.015 \pm 0.012$ & 0.78 & 5 (tilted) \\
\hline
\end{tabular}

Table S2. Anisotropy in Brillouin maps of the porcine cornea ex vivo. Please refer to Fig. 6. Corneal radius of curvature (ROC) calculated using corneal surface coordinates, fitted value \pm confidence interval for each parameter, coefficient of determination $\left(R^{2}\right)$, and sample orientation for the 5 ex vivo porcine corneas measured. The $\delta$ parameter was constrained to $0<\delta<0.25 \alpha_{x x}^{\text {(stroma) }}$. * indicates $\delta$ at bound. 


\section{Table of fit parameters for the human subjects}

\begin{tabular}{|c|c|c|c|c|}
\hline$\Omega(0)$ & $\alpha_{x x}^{\text {(stroma) }}$ & $\delta$ & $R^{2}$ & Fixation angle \\
\hline $5.717 \pm 0.003$ & $0.027 \pm 0.021$ & $0.009^{*}$ & 0.09 & $0^{\circ}$ \\
$5.697 \pm 0.005$ & $0.064 \pm 0.065$ & $0.015 \pm 0.029$ & 0.46 & $\sim 20^{\circ}$ \\
$5.696 \pm 0.003$ & $0.070 \pm 0.012$ & $0.023^{*}$ & 0.60 & $\sim 20^{\circ}$ \\
$5.700 \pm 0.004$ & $0.052 \pm 0.010$ & $0.017^{*}$ & 0.54 & $\sim 20^{\circ}$ \\
\hline
\end{tabular}

Table S3. Anisotropy of the human corneas in vivo. Please refer to Fig. 5. Fitted value \pm confidence interval for each parameter, coefficient of determination $\left(R^{2}\right)$, and eye fixation angle (relative to laser) for the 4 human subjects measured. A corneal radius of curvature (ROC) of $7.8 \mathrm{~mm}$ was used in calculating the beam incidence angles. The $\delta$ parameter was constrained to $0<\delta<0.25 \alpha_{x x}^{\text {(stroma) }}$. * indicates $\delta$ at bound. 